\title{
LINEAR DIFFERENCE EQUATIONS AND THEIR ANALYTIC SOLUTIONS*
}

BY

R. D. CARMICHAEL

Introduction.

The first existence theorem for difference equations in which analytic solutions were treated was obtained by GuICHARD $\dagger$ who, in a paper published in 1887, proved that if $\phi(x)$ is any entire function whatever there exists another entire function $f(x)$ satisfying the relation

$$
f(x+1)-f(x)=\phi(x) .
$$

Demonstrations of this theorem have also been given by APPELL $\ddagger$ and Hurwitr. $\S$ HuRwitz further showed how to solve the equation

$$
\phi(x) f(x+1)-\chi(x) f(x)=\psi(x),
$$

where $\phi(x), \chi(x), \psi(x)$ are meromorphic functions.

MFLLIN $\|$ has considered the nature of the solution of

$$
f(x+1)-r(x) f(x)=s(x)
$$

where $r(x)$ and $s(x)$ are rational functions.

BARNes $\$ has contributed an interesting study of the equation

$$
\phi(x) f(x+\omega)-\chi(x) f(x)=\psi(x),
$$

where $\omega$ is any constant different from zero and $\phi(x), \chi(x), \psi(x)$ are entire functions.

W ATson ** has considered the equation of the second order

$$
A(x) f(x+1)-B(x) f(x)+C(x) f(x-1)=0,
$$

* Presented to the Society September 6, 1910.

† Annales de i'École Normale supérieure, ser. 3, vol. 4 (1887), p. 361.

$\ddagger$ Journal de Mathématiques, ser. 4, vol. 7 (1891), p. 157, especially chapter I, pp. 159-176.

\$ Acta Mathematica, vol. 20 (1897), p. 285.

UActa Mathematica, vol. 15 (1891), p. 317.

Tl Proceedings of the London Mathematical Society, ser. 2, vol. 2 (1905), p. 438.

** Proceedings of the London Mathematical Society, ser. 2, vol. 8 (1910), p. 125. 
with some restrictions on $A(x), B(x), C(x)$, the object of his investigation being to obtain a solution which is of use in applications to the theory of linear differential equations. Two analytic solutions are obtained, but the question of their independence is left unanswered.

Finally, GaLbrun * has employed the Laplace transformation which carries a linear difference equation with polynomial coefficients into a differential equation, and by means of this transformation has obtained important existence theorems for the case in which the difference equation has polynomial coefficients.

The primary object of the present paper is to prove that there exists a fundamental system of simple analytic solutions of the system of $n$ linear homogeneous difference equations

$$
G_{i}(x+1)=x^{\alpha} \sum_{j=1}^{n} a_{i j}(x) G_{j}(x) \quad(i=1, \cdots, n),
$$

the known quantities entering into the equations being defined as follows :

1) $\alpha$ is a constant ;

2) the functions $a_{i j}(x)$ are single-valued and

$$
a_{i j}(x)=a_{i j}+a_{i j}^{\prime} x^{-1}+a_{i j}^{\prime \prime} x^{-2} \ldots \quad(i, j=1, \cdots, n) \quad|x|>R^{\prime} ;
$$

3) the constants $a_{i j}$ are such that the roots of the equation

$$
\left|a_{i j}-\delta_{i j} \rho\right|=0, \quad \delta_{i j}=\left\{\begin{array}{l}
0 \text { if } i \neq j, \\
1 \text { if } i=j,
\end{array}\right.
$$

are in absolute value different from each other and from zero.

In $\S 1$ a form of the method of successive approximation is developed in connection with a single equation of the first order and by means of it a study is made of the nature of a simple particular solution of the equation.

The methods of $\S 1$ are readily extended in $\S 2$ in such a way that they lead to a similar solution of the normal system

$$
f_{i}(x+1)-a_{i}\left(1+\frac{1}{x}\right)^{m_{i}} f_{i}(x)=\sum_{j=1}^{n} \phi_{i j}(x) f_{j}(x) \quad(i=1, \cdots, n),
$$

where

and

$$
0<\left|a_{1}\right|<\left|a_{2}\right|<\cdots<\left|a_{n}\right|
$$

$$
\phi_{i j}(x)=\phi_{i j}^{\prime \prime} x^{-2}+\phi_{i j}^{\prime \prime \prime} x^{-3}+\cdots \quad(i, j=1, \cdots, n) \quad|x| \geqq R>1 .
$$

A method of induction in $\S 3$ leads to the determination of a fundamental system of solutions of $(B)$ and to the development of important properties of the functions of which it is composed.

In $\S 4$ it is shown that system $(A)$ can be reduced to the normal form $(B)$

*Comptes rend us, vol. 148 (1909), p. 905 ; vol. 149 (1909), p. 1046 ; vol. 150 (1910), p. 206 ; vol. 151 (1910), p. 1114. 
and thus the general existence theorem for $(A)$ is obtained. Finally, an application of this result is made in deriving the general existence theorem for a single equation of the $n$th order.

I desire to express my cordial thanks to Professor G. D. Birkhoff for valuable suggestions and encouragement in the preparation of the paper.

\section{§1. The method of successive approximation for a single homogeneous equation of the first order.}

We shall begin by considering a single homogeneous equation of the first order in the normal form *

$$
f(x+1)-a\left(1+\frac{1}{x}\right)^{m} f(x)=\phi(x) f(x), \quad a \neq 0,
$$

or

where

$$
f(x+1)=\psi(x) f(x), \quad \psi(x) \equiv a\left(1+\frac{1}{x}\right)^{m}+\phi(x),
$$

$$
\phi(x)=\phi^{\prime \prime} x^{-2}+\phi^{\prime \prime \prime} x^{-3}+\cdots, \quad \text { for }|x| \geqq R>1 .
$$

We assume that $\psi(x)$ is single-valued and analytic at all points of the complex plane where it is defined, and that that determination of $(1+1 / x)^{m}$ is taken which approaches 1 as $x$ approaches infinity. The sequence of equations

$$
\begin{aligned}
& f^{(1)}(x+1)-a\left(1+\frac{1}{x}\right)^{m} f^{(1)}(x)=0 \\
& f^{(2)}(x+1)-a\left(1+\frac{1}{x}\right)^{m} f^{(2)}(x)=\phi(x) f^{(1)}(x), \\
& f^{(3)}(x+1)-a\left(1+\frac{1}{x}\right)^{m} f^{(3)}(x)=\phi(x) f^{(2)}(x),
\end{aligned}
$$

will serve to define a sequence of approximation functions $f^{(1)}(x), f^{(2)}(x), \ldots$ A particular solution of the first equation is evidently $a^{x} x^{m}$. All the other equations of the set have the general non-homogeneous form

$$
g(x+1)-a\left(1+\frac{1}{x}\right)^{m} g(x)=\eta(x) .
$$

* It is easy to reduce the general first order equation

$$
G(x+1)=x^{a}\left(\theta+\theta^{\prime} x^{-1}+\theta^{\prime \prime} x^{-2}+\cdots\right) G(x), \quad \theta \neq 0,
$$

to this form. Put

$$
G(x)=\{\Gamma(x)\}^{a} f(x) .
$$

Then

$$
f(x+1)=\left(\theta+\theta^{\prime} x^{-1}+\theta^{\prime \prime} x^{-2}+\cdots\right) f(x) ;
$$

and for $|x|$ sufficiently large this evidently may be put in the form (1). 
One readily obtains a formal solution of this equation as follows : Let

$$
g(x)=a^{x} x^{m} \bar{g}(x),
$$

where $a^{x} x^{m}$ has any determination fixed by making a cut along the negative axis of reals in the $x$-plane. Substituting in (4) this value of $g(x)$ and dividing by $a^{x+1}(x+1)^{m}$ one has

$$
\bar{g}(x+1)-\bar{g}(x)=\frac{\eta(x)}{a^{x+1}(x+1)^{m}},
$$

an equation which has the formal solution

Hence

$$
\bar{g}(x)=-\frac{\eta(x)}{a^{x+1}(x+1)^{m}}-\frac{\eta(x+1)}{a^{x+2}(x+2)^{m}}-\frac{\eta(x+2)}{a^{x+3}(x+3)^{m}}-\cdots
$$

Now

$$
g(x)=-\frac{\eta(x)}{a\left(1+\frac{1}{x}\right)^{m}}-\frac{\eta(x+1)}{a^{2}\left(1+\frac{2}{x}\right)^{m}}-\frac{\eta(x+2)}{a^{3}\left(1+\frac{3}{x}\right)^{m}}-\cdots
$$

where

$$
a^{r}\left(1+\frac{r}{x}\right)^{m}=\lambda(x) \lambda(x+1) \cdots \lambda(x+r-1),
$$

$$
\lambda(x)=a\left(1+\frac{1}{x}\right)^{m}
$$

and therefore (5) may be written in the form*

$$
g(x)=-\sum_{i=0}^{\infty} \frac{\eta(x+i)}{\lambda(x) \lambda(x+1) \cdots \lambda(x+i)} .
$$

This yields not merely a formal but an actual solution for any values of $x$ for which it converges; and if $\eta(x)$ is analytic, then (by Weierstrass's theorem) it yields an analytic solution throughout any two-dimensional continuum where it converges uniformly.

By the aid of $(6)$ we obtain a sequence of formal solutions $f^{(i)}(x)$ satisfying (3) identically. Employing the notation

$$
S_{x}(\chi)=-\sum_{i=0}^{\infty} \frac{\chi(x+i)}{\lambda(x)} \lambda \frac{\lambda(x+1) \cdots \lambda(x+i)}{\lambda(x+1)}
$$

and using for each equation that particular formal solution which is obtained by adding the solution $a^{x} x^{m}$ of the homogeneous equation to the solution derived from $(6)$, we have

$$
\begin{aligned}
& f^{(1)}(x)=a^{x} x^{m}, \\
& f^{(2)}(x)=a^{x} x^{m}+S_{x}\left(\phi a^{x} x^{m}\right), \\
& f^{(3)}(x)=a^{x} x^{m}+S_{x}\left\{\phi a^{x} x^{m}+\phi S_{x}\left(\phi a^{x} x^{m}\right)\right\},
\end{aligned}
$$

* Cf. Mellin, Acta Mathematica, vol. 15 (1891), p. 319. 
It is easy to see that this leads to a formal solution $f_{1}(x)$ of $(1)$, defined by the series

$$
\begin{aligned}
f_{1}(x)=a^{x} x^{m}+S_{x}\left(\phi a^{x} x^{m}\right)+S_{x}\{ & \left.\phi S_{x}\left(\phi a^{x} x^{m}\right)\right\} \\
& +S_{x}\left[\phi S_{x}\left\{\phi S_{x}\left(\phi a^{x} x^{m}\right)\right\}\right]+\cdots \cdot
\end{aligned}
$$

That this series formally satisfies (1) we may verify by forming the difference $f_{1}(x+1)-a(1+1 / x)^{m} f_{1}(x)$ and effecting a reduction of the resulting series by means of the general formula

$$
S_{x+1}(\chi)-a\left(1+\frac{1}{x}\right)^{m} S_{x}(\chi)=\chi
$$

Consequently whenever all the series $S_{x}$ in the terms of (7) and also the series (7) itself converge, this series represents a solution of (1).

In studying the nature of the formal solution (7) (and in the work of the succeeding sections also) it will be convenient to have names assigned to certain regions of the plane about which we shall have to speak often. Let $R$ be the

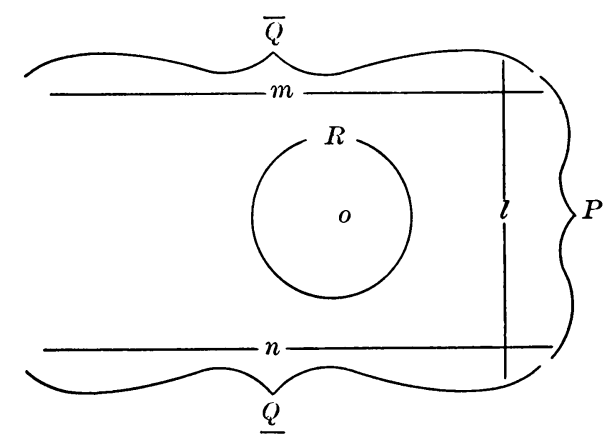

FIG. 1.

circle $|x|=R$. Let $l$ be a line cutting the positive axis of reals at right angles and not cutting $R$. The part of the finite plane to the right of and including $l$ will be called a $P$-region. Let $m$ and $n$ be parallel to the axis of reals, $m$ lying above and $n$ below $R$. The part of the finite plane lying above and including $m$ will be named a $\bar{Q}$-region; that lying below and including $n$, a $Q$-region; these two together, a $Q$-region. A region formed by the combination of the $P$ - and $Q$-regions will be called a $D$-region. The part of the finite plane not included within $D$ will be called the $E$-region corresponding to $D$. It is scarcely necessary to remark that $\bar{Q}$ and $\underline{Q}$ have each a part in common with $P$.

In what follows immediately we are to prove the existence of $f_{1}(x)$ and develop its properties in particular regions $P_{1}, Q_{1}, D_{1}, E_{1}$; for convenience all these regions are defined at once. For any positive constant $c$, less than $|a|$, there 
exists an $R^{\prime}$ such that

$$
\left|a\left(1+\frac{1}{x}\right)^{m}\right|>c, \quad \text { for }|x|>R^{\prime} .
$$

From the nature of the function $\phi(x)$ it follows that there exists an $M$ such that

If one writes

$$
|\phi(x)|<\frac{M c}{|x|^{2}}, \quad \text { for }|x| \geqq R .
$$

$$
x=u \pm v \sqrt{-1}
$$

where $v$ is positive and both $u$ and $v$ are real, it is now clear that one may choose particular regions $P, Q, D, E$ - call them $P_{1}, Q_{1}, D_{1}, E_{1}$ respectively-such that

$$
\begin{gathered}
|\phi(x)|<\frac{M c}{|x|^{2}}, \quad\left|a\left(1+\frac{1}{x}\right)^{m}\right|>c, \quad \text { for } x \text { in } D_{1} ; \\
u>M+1, \quad \text { for } x \text { in } P_{1} ; \\
\frac{A}{v}<\frac{M \pi}{v}+\frac{2 M}{v^{2}}<1, \quad \text { for } x \text { in } Q_{1}, A \text { being a constant; } \\
a\left(1+\frac{1}{x}\right)^{m}+\phi(x) \neq 0, \quad \text { for } x \text { in } D_{1} .
\end{gathered}
$$

Let us consider the function

$$
\bar{S}_{x}\left(\phi a^{x} x^{m}\right) \equiv \sum_{i=0}^{\infty}\left|\frac{\phi(x+i) a^{x+i}(x+i)^{m}}{\lambda(x) \lambda(x+1) \cdots \lambda(x+i)}\right|=\sum_{i=0}^{\infty}\left|\frac{\phi(x+i) a^{x} x^{m}}{\lambda(x+i)}\right|
$$

Employing inequalities (8) one has the relation*

$$
\sum_{i=0}^{\infty}\left|\frac{\phi(x+i) a^{x} x^{m}}{\lambda(x+i)}\right| \ll \sum_{i=0}^{\infty} \frac{M\left|a^{x} x^{m}\right|}{(u+i)^{2}+v^{2}}
$$

subsisting throughout $D_{1}$. The series of the last member of (13) is evidently uniformly convergent when $x$ lies in any closed region in $D_{1}$. The same then is true of the series in the first member, and hence also of the series in (12). Therefore $S_{x}\left(\phi a^{x} x^{m}\right)$ is a uniformly convergent series in the same closed region and hence, in accordance with a well-known theorem of Weierstrass, represents in this region an analytic function of $x$. From this it follows that the first and second terms of the series in (7) represent functions which are analytic in $D_{1}$.

In order to show that the remaining terms are analytic in the same region it is necessary to know a bound to the magnitude of $\left|S_{x}\left(\phi a^{x} x^{m}\right)\right|$ as $x$ varies. In carrying out the needful reckoning it is convenient to treat the regions $P_{1}$ and $Q_{1}$ separately.

\footnotetext{
* The symbol 《 is to be read is less term by term than.
} 
First, let us consider the region $P_{1}$. Of the two series

$$
\sum_{i=0}^{\infty} \frac{M}{(u+i)^{2}+v^{2}}, \quad \sum_{i=0}^{\infty} \frac{M}{(u+i)^{2}}, \quad \text { for } x \text { in } P_{1},
$$

the terms of the first in order are equal to or less than the corresponding terms. of the second. But the latter series is less than the integral

and therefore

$$
\int_{u-1}^{\infty} \frac{M}{z^{2}} d z=\frac{M}{u-1}
$$

$$
\bar{S}_{x}\left(\phi a^{x} x^{m}\right)-\left|a^{x} x^{m}\right| \frac{M}{u-1}
$$

Hence

$$
\left|S_{x}\left(\phi a^{x} x^{m}\right)\right|<\left|a^{x} x^{m}\right| \frac{M}{u-1}
$$

Using this result and making an argument similar to that associated with inequality (13) one may show that $S_{x}\left\{\phi S_{x}\left(\phi a^{x} x^{m}\right)\right\}$ is analytic throughout $P_{1}$. Again, in view of (14) we have

$\bar{S}_{x}\left\{\phi \bar{S}_{x}\left(\phi a^{x} x^{m}\right)\right\}<\bar{S}_{x}\left\{\phi\left|a^{x} x^{m}\right| \frac{M}{u-1}\right\}<\frac{M}{u-1} \bar{S}_{x}\left(\phi a^{x} x^{m}\right)<\left|a^{x} x^{m}\right| \frac{M^{2}}{(u-1)^{2}}$.

Similarly it may be proved that $S_{x}\left[\phi S_{x}\left\{\phi S_{x}\left(\phi a^{x} x^{m}\right)\right\}\right]$ is analytic in $P_{1}$; an upper bound for the value of $\bar{S}_{x}^{x}\left[\phi \bar{S}_{x}^{x}\left\{\phi \bar{S}_{x}\left(\phi a^{x} x^{m}\right)\right\}\right]$ may be obtained; and so on. Hence in series (7) every term is analytic throughout $P_{1}$; moreover the following inequality has been established for $x$ in $P_{1}$ :

$$
\begin{aligned}
\bar{S}_{x}\left(\phi a^{x} x^{m}\right)+\bar{S}_{x}\left\{\phi \bar{S}_{x}\left(\phi a^{x} x^{m}\right)\right\}+\bar{S}_{x}\left[\phi \bar{S}_{x}\left\{\phi \bar{S}_{x}\left(\phi a^{x} x^{m}\right)\right\}\right]+\cdots \\
\ll \frac{M\left|a^{x} x^{m}\right|}{u-1}+\frac{M^{2}\left|a^{x} x^{m}\right|}{(u-1)^{2}}+\frac{M^{3}\left|a^{x} x^{m}\right|}{(u-1)^{3}}+\cdots
\end{aligned}
$$

From (9) it follows that the second member of (15) is absolutely and uniformly convergent throughout any closed region in $P_{1}$; and therefore the same is true of the first member. Hence series ( 7 ) is uniformly and absolutely convergent throughout this region. But its terms are analytic functions of $x$; and therefore, by Weierstrass's theorem, its sum $f_{1}(x)$ is an analytic function of $x$ throughout $P_{1}$. Evidently, then, $f(x)=f_{1}(x)$ is a solution of (1).

A comparison of (7) and (15) shows that the following inequalities are valid. for $x$ in $P_{1}$ :

$$
\begin{aligned}
&\left|a^{x} x^{m}\right|\left\{1-\frac{M}{u-1}-\frac{M^{2}}{(u-1)^{2}}-\cdots\right\}<\left|f_{1}(x)\right| \\
&<\left|a^{x} x^{m}\right|\left\{1+\frac{M}{u-1}+\frac{M^{2}}{(u-1)^{2}}+\cdots\right\} .
\end{aligned}
$$


Hence we have

$$
\left|a^{x} x^{m}\right| \frac{u-2 M-1}{u-M-1}<\left|f_{1}(x)\right|<\left|a^{x} x^{m}\right| \frac{u-1}{u-M-1},
$$

a set of fundamental inequalities bounding the increase or decrease of $f_{1}(x)$ throughout $P_{1}$.

Again using relation (13) as a point of departure, we obtain similar results valid in $Q_{1}$. In this region $v$ is different from zero. It is evident that

$$
\sum_{i=0}^{\infty} \frac{M}{(u+i)^{2}+v^{2}}<\sum_{i=-\infty}^{\infty} \frac{M}{(u+i)^{2}+v^{2}} \leqq 2\left\{M \int_{0}^{\infty} \frac{d z}{z^{2}+v^{2}}+\frac{M}{v^{2}}\right\}=\frac{M \pi}{v}+\frac{2 M}{v^{2}}<\frac{A}{v},
$$

$A$ being a properly chosen constant. Comparing this result with (12) and (13) one has

$$
\left|S_{x}\left(\phi a^{x} x^{m}\right)\right| \leqq \bar{S}_{x}\left(\phi a^{x} x^{m}\right)<\left|a^{x} x^{m}\right| \frac{A}{v}
$$

On account of (10) and (18) it is clear that one may employ an argument similar to that associated with (13) to show that $S_{x}\left\{\phi S_{x}\left(\phi a^{x} x^{m}\right)\right\}$ is an analytic function of $x$ in $Q_{1}$. Again, on account of (17.) one has

$$
\bar{S}_{x}\left\{\phi \bar{S}_{x}\left(\phi a^{x} x^{m}\right)\right\}<\bar{S}_{x}\left\{\phi\left|\boldsymbol{a}^{x} x^{m}\right| \frac{A}{v}\right\}=\frac{A}{v} \bar{S}_{x}\left(\phi a^{x} x^{m}\right)<\left|a^{x} x^{m}\right| \frac{A^{2}}{v^{2}}
$$

In like manner it may be shown that the function $S_{x}\left[\phi S_{x}\left\{\phi S_{x}\left(\phi a^{x} x^{m}\right)\right\}\right]$ is analytic throughout $Q_{1}$; also, an upper bound for the value of

$$
\bar{S}_{x}\left[\phi \bar{S}_{x}\left\{\phi \bar{S}_{x}\left(\phi a^{x} x^{m}\right)\right\}\right]
$$

may be obtained; and so on. Hence every term in series (7) is analytic in $Q_{1}$. Moreover, for this region the following relation exists :

$$
\begin{gathered}
\bar{S}_{x}\left(\phi a^{x} x^{m}\right)+\bar{S}_{x}\left\{\phi \bar{S}_{x}\left(\phi a^{x} x^{m}\right)\right\}+\bar{S}_{x}\left[\phi \bar{S}_{x}\left\{\phi \bar{S}_{x}\left(\phi a^{x} x^{m}\right)\right\}\right]+\cdots \\
\ll\left|a^{x} x^{m}\right| \frac{A}{v}+\left|a^{x} x^{m}\right| \frac{A^{2}}{v^{2}}+\left|a^{x} x^{m}\right| \frac{A^{3}}{v^{3}}+\cdots
\end{gathered}
$$

By a method similar to that employed in the previous case it is now easy to show that $f_{1}(x)$ is analytic in $Q_{1}$, and that throughout this region it is bounded as in the inequalities

$$
\left|a^{x} x^{m}\right| \frac{v-2 A}{v-A}<\left|f_{1}(x)\right|<\left|a^{x} x^{m}\right| \frac{v}{v-A}
$$

It remains to determine the nature of $f_{1}(x)$ in the region $E_{1}$. For this study series (7) does not furnish a convenient means. We proceed as follows: If we solve (1) for $f(x)$ and in the result write $x-1$ for $x$ we have

$$
f(x-1)=\bar{\psi}(x-1) f(x)
$$


where

$$
\bar{\psi}(x)=\{\psi(x)\}^{-1} \text {. }
$$

By means of (21), the solution $f_{1}(x)$ which is known to exist in $P_{1}$ can be extended across the plane to the left; and in this way the nature of $f_{1}(x)$ in $E_{1}$ is determined.

Consider the set of points $[S]$ which is formed of the set $[\alpha]$ and of the sets $[\alpha-i], i=1,2,3, \ldots$, where $[\alpha]$ is the set of singularities of $\bar{\psi}(x)$. A point $\alpha-i$ is said to be congruent to $\alpha$ on the left; in a similar way congruence on the right is defined. It is easy to see that $f_{1}(x)$ is analytic at every point $x^{\prime}$ not belonging to $[S]$. For, by (21),

$$
f_{1}\left(x^{\prime}\right)=\bar{\psi}\left(x^{\prime}\right) \bar{\psi}\left(x^{\prime}+1\right) \cdots \bar{\psi}\left(x^{\prime}+\nu-1\right) f_{1}\left(x^{\prime}+\nu\right) \text {. }
$$

$\nu$ can be so chosen that $x^{\prime}+\nu$ lies in $P_{1}$. For such a value of $\nu, f_{1}(x)$ is analytic at $x^{\prime}+\nu$; also, $\bar{\psi}(x)$ is analytic at each of the points $x^{\prime}, x^{\prime}+1, \ldots$, $x^{\prime}+\nu-1$. Therefore $f_{1}(x)$ is analytic at $x^{\prime}$. Combining this with previous results, we see that $f_{1}(x)$ is analytic throughout the finite plane except at the singularities [ $\alpha]$ of $\bar{\psi}(x)$ and the points congruent to them on the left; at these points its character depends upon the nature of the particular $\bar{\psi}(x)$ involved.

Let each point of $[S]$ be inclosed by a small circle of radius $\epsilon$ having the point as its center. Denote by $E_{1 \epsilon}$ the whole of that part of $E_{1}$ no point of which is within one of these small circles.

Inequalities bounding the increase or decrease of $f_{1}(x)$ for $x$ in $E_{1 \mathrm{e}}$ may be found as follows: Consider any positive constants $a_{-}$and $a_{+}$satisfying the relations

Since

$$
a_{-}<|a|<a_{+} .
$$

$$
\underset{x=\infty}{L}\left\{a\left(1+\frac{1}{x}\right)^{m}+\phi(x)\right\}=a,
$$

it is clear that $r$ may be chosen so that

$$
a_{+}>\left|a\left(1+\frac{1}{x}\right)^{m}+\phi(x)\right|>a_{-}, \text {for }|x|>r,
$$

and also so that the circle $|x|=r$ cuts the boundary of $E_{1}$ twice on the left of the origin and at no other point. Since $f_{1}(x)$ is everywhere analytic in $E_{1 \epsilon}$ it follows that there exist constants $\bar{N}_{1}$ and $\bar{N}_{2}$ such that

$$
N_{1} \leqq\left|f_{1}\left(x^{\prime \prime}\right)\right|<\bar{N}_{2}, \quad \text { for } x^{\prime \prime} \text { in } E_{1 \mathrm{e}} \text { and } r \leqq\left|x^{\prime \prime}\right| \leqq r+1 \text {. }
$$

But (21) yields the equation

$$
f_{1}\left(x^{\prime \prime}-\mu\right)=\bar{\psi}\left(x^{\prime \prime}-\mu\right) \bar{\psi}\left(x^{\prime \prime}-\mu+1\right) \cdots \psi\left(x^{\prime \prime}-1\right) f_{1}\left(x^{\prime \prime}\right) .
$$


Hence we have

$$
\bar{N}_{1} a_{+}^{-\mu} \leqq\left|f\left(x^{\prime \prime}-\mu\right)\right|<\bar{N}_{2} a_{-}^{-\mu}, \quad \text { for } x^{\prime \prime} \text { in } E_{1 \mathrm{e}} \text { and } r \leqq\left|x^{\prime \prime}\right| \leqq r+1 \text {. }
$$

As $\mu$ varies over the range $\mu=0,1,2, \ldots, x^{\prime \prime}-\mu$ runs over all points in $E_{1 e}$ and outside of the circle $|x|=r$. Also, $f_{1}(x)$ is finite in the part of $E_{1}$ which is in the circle $|x|=r$. Hence it is clear that there exist constants $N_{1}$ and $N_{2}$ such that

$$
N_{1}\left|a_{+}^{x}\right| \leqq\left|f_{1}(x)\right|<N_{2}\left|a_{-}^{x}\right|, \quad \text { for } x \text { in } E_{1 \epsilon},
$$

the result sought.

So far we have said nothing of the position of the zeros of $f_{1}(x)$. By means of equation (1) we shall show that they can occur only at the singularities of $\psi(x)$ and at points congruent to them on the left. We have

$$
f_{1}\left(x^{\prime}+\mu\right)=\psi\left(x^{\prime}+\mu-1\right) \psi\left(x^{\prime}+\mu-2\right) \cdots \psi\left(x^{\prime}\right) f_{1}\left(x^{\prime}\right),
$$

where $\mu$ is a positive integer. From inequality (16) it follows that for every $x^{\prime}$ there exists a $\mu$ such that $f_{1}\left(x^{\prime}+\mu\right)$ is different from zero. Hence $f_{1}\left(x^{\prime}\right)$ can be zero only when one at least of the factors $\psi\left(x^{\prime}\right), \psi\left(x^{\prime}+1\right), \cdots, \psi\left(x^{\prime}+\mu-1\right)$ is infinite: and therefore the zeros of $f_{1}(x)$ must all be contained in the set of points composed of the singularities of $\psi(x)$ and the points congruent to them on the left.

The principal results contained in the section may be enunciated as follows : The difference equation

$f(x+1)=\psi(x), f(x)$ or $f(x-1)=\bar{\psi}(x-1) f(x), \quad \bar{\psi}(x) \equiv\{\psi(x)\}^{-1}$, in which $\psi(x)$ is single-valued and

$\psi(x)=a\left(1+\frac{1}{x}\right)^{m}+\phi(x), \quad \phi(x)=\phi^{\prime \prime} x^{-2}+\phi^{\prime \prime \prime} x^{-3}+\cdots$, for $|x| \geqq R>1$,

has a particular solution $f_{1}(x)$ which is analytic throughout the finite plane except at the singularities [ $\alpha]$ of $\bar{\psi}(x)$ and the points congruent to them on the left, and which can vanish only at the singularities [ $\beta]$ of $\psi(x)$ and at points congruent to them on the left. If $D_{1}$ is defined by relations (8) to (11), then throughout any closed region in $D_{1}$ this solution is representable as a uniformly converging series in the form

$f_{1}(x)=a^{x} x^{m}+S_{x}\left(\phi a^{x} x^{m}\right)+S_{x}\left\{\phi S_{x}\left(\phi a^{x} x^{m}\right)\right\}+S_{x}\left[\phi S_{x}\left\{\phi S_{x}\left(\phi a^{x} x^{m}\right)\right\}\right]+\cdots$

The increase or decrease of $f_{1}(x)$ for $x$ in the corresponding regions $P_{1}$ and $Q_{1}$ is bounded as in the inequalities

$$
\begin{gathered}
\left|a^{x} x^{m}\right| \frac{u-2 M-1}{u-M-1}<\left|f_{1}(x)\right|<\left|a^{x} x^{m}\right| \frac{u-1}{u-M-1}, \text { for } x \text { in } P_{1} \\
\left|a^{x} x^{m}\right| \frac{v-2 A}{v-A}<\left|f_{1}(x)\right|<\left|a^{x} x^{m}\right| \frac{v}{v-A}, \text { for } x \text { in } Q_{1} .
\end{gathered}
$$


Also, for any positive constants $a_{-}$and $\alpha_{+}$satisfying the relations

$$
a_{-}<|a|<a_{+}
$$

there exist other constants $N_{1}, N_{2}$ such that

$$
N_{1}\left|a_{+}^{x}\right| \leqq\left|f_{1}(x)\right|<N_{2}\left|a_{-}^{x}\right|, \text { for } x \text { in } E_{1 \mathrm{e}} \text {. }
$$

Remark. About each statement in the conclusion of this theorem it should be observed that, insofar as the statement refers to the region $D_{1}$ or any part of it, it is independent of the nature of $\phi(x)$ in the region exterior to $D_{1}$. More explicitly: If $\phi(x)$ is any function which is analytic in some D-region and if also

$$
|\phi(x)|<\frac{M c}{|x|^{2}}, \text { for } x \text { in } D,
$$

then there exist regions $P_{1}, Q_{1}, D_{1}$ having as before the properties (8) to (10), and there is a particular solution $f_{1}(x)$ of the difference equation having in $D_{1}$ the same properties as the solution $f_{1}(x)$ of the equation in the theorem. These conclusions follow from the preceding discussion without change of argument.

§2. The method of successive approximation for a normal system of $n$ equations. Properties of the solution thus obtained.

Let us consider the system of $n$ linear difference equations of the first order in the normal form

$$
f_{i}(x+1)-a_{i}\left(1+\frac{1}{x}\right)^{m_{i}} f_{i}(x)=\sum_{j=1}^{n} \phi_{i j}(x) f_{j}(x) \quad(i=1, \cdots, n)
$$

or where

$$
f_{i}(x+1)=\sum_{j=1}^{n} \psi_{i j}(x) f_{j}(x), \quad \psi_{i j}(x) \equiv \delta_{i j} a_{i}\left(1+\frac{1}{x}\right)^{m_{i}}+\phi_{i j}(x),
$$

$$
0<\left|a_{1}\right|<\left|a_{2}\right|<\cdots<\left|a_{n}\right|
$$

and

(26) $\phi_{i j}(x)=\phi_{i j}^{\prime \prime} x^{-2}+\phi_{i j}^{\prime \prime \prime} x^{-3}+\cdots \quad(i, j=1, \cdots, n)$, for $|x| \geqq R>1$.

We assume that $\psi_{i j}(x)$ is single-valued. If we solve (24) for $f_{i}(x)$ in terms of $f_{i}(x+1), i=1, \cdots, n$, and in the result write $x-1$ for $x$, we obtain

$$
f_{i}(x-1)=\sum_{j=1}^{n} \bar{\psi}_{i j}(x-1) f_{j}(x) \quad(i=1, \cdots, n),
$$

$\bar{\psi}_{i j}(x)$ having the form

$$
\bar{\psi}_{i j}(x)=\frac{\alpha_{i j}(x)}{\Delta}
$$

where $\Delta$ is the determinant

$$
\Delta \equiv\left|\delta_{i j} a_{i}\left(1+\frac{1}{x}\right)^{m_{i}}+\phi_{i j}(x)\right|
$$


and $\alpha_{i j}(x)$ is the cofactor of the element in the $j$ th column and the ith row of $\Delta$.

The object of this section is to obtain a particular solution of (24) and to study its properties throughout the plane.

On account of (25) and (26) it is clear that there exist particular regions $P_{1}$, $Q_{1}, D_{1}, E_{1}$ and constants $c, M$ and $A$ such that the following inequalities are true :

$$
\begin{gathered}
\left|\phi_{i j}(x)\right|<\frac{M c}{n|x|^{2}}, \quad\left|a_{i}\left(1+\frac{1}{x}\right)^{m_{i}}\right|>c \quad(i, j=1, \cdots, n), \text { for } x \text { in } D_{1} \\
\left|a_{1}\left(1+\frac{1}{x}\right)^{m_{1}}\right|<\left|a_{i}\left(1+\frac{1}{x}\right)^{m_{i}}\right| \quad(i=2, \cdots, n), \text { for } x \text { in } D_{1} \\
u>M+1, \quad \text { for } x \text { in } P_{1}, \quad \text { where } x=u \pm v \sqrt{-1} \\
\frac{A}{v}<\frac{M \pi}{v}+\frac{2 M}{v^{2}}<1, \text { for } x \text { in } Q_{1} \\
\Delta \neq 0, \text { for }|x| \geqq \bar{R} \geqq R
\end{gathered}
$$

In the study of our system of equations these regions are treated separately.

It will be convenient to employ the notation

$$
S_{x i}(\chi) \equiv-\sum_{\nu=0} \frac{\chi(x+\nu)}{\lambda_{i}(x) \lambda_{i}(x+1) \cdots \lambda_{i}(x+\nu)}, \quad \lambda_{i}(x) \equiv a_{i}\left(1+\frac{1}{x}\right)^{m_{i}}
$$

also we write

$$
u_{i} \equiv C_{i} a_{i}^{x} x^{m_{i}}
$$

where $C_{i}$ is any periodic function of $x$ of period 1 and analytic throughouc. $D_{1}$.

The form of the method of successive approximation employed in the preceding section is readily generalized so as to lead to a particular solution of (24). Consider the set of systems each of $n$ linear equations, all except the first beingnon-homogeneous :

$$
\begin{aligned}
& f_{i}^{(1)}(x+1)-a_{i}\left(1+\frac{1}{x}\right)^{m_{i}} f_{i}^{(1)}(x)=0 \\
& f_{i}^{(2)}(x+1)-a_{i}\left(1+\frac{1}{x}\right)^{m_{i}} f_{i}^{(2)}(x)=\sum_{j=1}^{n} \phi_{i j}(x) f_{j}^{(1)}(x), \quad(i=1, \cdots, n) . \\
& f_{i}^{(3)}(x+1)-a_{i}\left(1+\frac{1}{x}\right)^{m_{i}} f_{i}^{(3)}(x)=\sum_{j=1}^{n} \phi_{i j}(x) f_{j}^{(2)}(x)
\end{aligned}
$$

Proceeding as in the foregoing section, one writes out in order formal solutions 
of these systems, obtaining thus the following set of approximation functions :

$f_{i}^{(1)}(x)=u_{i}$.

$f_{i}^{(2)}(x)=u_{i}+S_{x i}\left(\sum_{j=1}^{n} \phi_{i j} u_{j}\right)$

$f_{i}^{(3)}(x)=u_{i}+S_{x i}\left(\sum_{j=1}^{n} \phi_{i j} u_{j}\right)+S_{x i}\left\{\sum_{j=1}^{n} \phi_{i j} S_{x j}\left(\sum_{k=1}^{n} \phi_{j k} u_{k}\right)\right\}$

$(i=1, \cdots, n)$.

It is easy to verify by actual substitution that a solution of system (24) is obtained in the limit functions (if they exist) of the sequences $f_{i}^{(1)}, f_{i}^{(2)}, f_{i}^{(3)}$, $\ldots, i=1, \ldots, n$; that is, in

$$
\begin{aligned}
f_{i}(x)=u_{i} & +S_{x i}\left(\sum_{j=1}^{n} \phi_{i j} u_{j}\right)+S_{x i}\left\{\sum_{j=1}^{n} \phi_{i j} S_{x j}\left(\sum_{k=1}^{n} \phi_{j k} u_{k}\right)\right\} \\
& +S_{x i}\left[\sum_{j=1}^{n} \phi_{i j} S_{x j}\left\{\sum_{k=1}^{n} \phi_{j k} S_{x k}\left(\sum_{l=1}^{n} \phi_{k l} u_{l}\right)\right\}\right]+\cdots
\end{aligned}
$$

This solution is in general illusory on account of the divergence of certain series contained in the terms of the expansions formally representing the $f$ 's. In fact, let $a_{r}$ and $a_{s}$ be any two $a$ 's such that $\left|a_{r}\right|>\left|a_{s}\right| \cdot$ Put

$$
C_{i}=0, i \neq r ; \quad C_{r}=1 \text {. }
$$

For $i=s$ the second term in the series in (35) becomes

$$
\begin{aligned}
S_{x \delta}\left(\phi_{s r} a_{r}^{x} x^{m_{r}}\right) & \equiv-\sum_{\nu=0}^{\infty} \frac{\phi_{s r}(x+\nu) a_{r}^{x+\nu}(x+\nu)^{m_{r}}}{\lambda_{s}(x) \cdots \lambda_{s}(x+\nu)} \\
& =-\sum_{\nu=0}^{\infty} \frac{\phi_{s r}(x+\nu) a_{r}^{x+\nu}(x+\nu)^{m_{r}}}{a_{s}^{x+\nu}(x+\nu)^{m_{s}}}
\end{aligned}
$$

a series which is evidently divergent at least for some values of $\phi_{s r}(x)$ as for instance $\phi_{s r}(x) \equiv 1 / x^{2}$.

On the other hand, there is at least one particular convergent solution, arising from the following special choice of values of the periodic functions $C_{i}$ :

$$
C_{i}=0, \quad i \neq 1 ; \quad C_{1}=1 .
$$

If we call this particular solution $f_{i 1}(x)$, we have formally from (35)

$$
\begin{aligned}
f_{i 1}(x)= & \delta_{i 1} a_{1}^{x} x^{m_{1}}+S_{x i}\left(\phi_{i 1} a_{1}^{x} x^{m_{1}}\right)+S_{x i}\left\{\sum_{j=1}^{n} \phi_{i j} S_{x j}\left(\phi_{j 1} a_{1}^{x} x^{m_{1}}\right)\right\} \\
& +S_{x i}\left[\sum_{j=1}^{n} \phi_{i j} S_{x j}\left\{\sum_{k=1}^{n} \phi_{j k} S_{x k}\left(\phi_{k 1} a_{1}^{x} x^{m_{1}}\right)\right\}\right]+\cdots \quad(i=1, \cdots, n) .
\end{aligned}
$$


That all the infinite series contained in (37) are actually convergent in $D_{1}$ is readily shown. Consider the series

$$
\begin{aligned}
\delta_{i 1} a_{1}^{x} x^{m_{1}}+\bar{S}_{x i}\left(\frac{M c}{n x^{2}} a_{1}^{x} x^{m_{1}}\right)+\bar{S}_{x i}\left\{\frac{M c}{n x^{2}} \sum_{j=1}^{n} \bar{S}_{x j}\left(\frac{M c}{n x^{2}} a_{1}^{x} x^{m_{1}}\right)\right\} \\
+\bar{S}_{x i}\left[\frac{M c}{n} \bar{x}^{2} \sum_{j=1}^{n} \bar{S}_{x j}\left\{\frac{M c}{n x^{2}} \sum_{k=1}^{n} \bar{S}_{x k}\left(\frac{M c}{n x^{2}} a_{1}^{x} x^{m_{1}}\right)\right\}\right]+\cdots,
\end{aligned}
$$

where $\bar{S}_{x i}$ denotes what $S_{x i}$ becomes when the terms of the series which it indicates are replaced by their absolute values. On account of the first inequality (30) it is evident that all the infinite series in (37) are convergent provided that all those in (38) are likewise convergent; for if these expansions are written out in detail, the former, term by term, is less in absolute value than the latter.

Compare (38) with the following series

$$
\begin{aligned}
a_{1}^{x} x^{m_{1}}+\bar{S}_{x 1}\left(\frac{M c}{x^{2}} a_{1}^{x} x^{m_{1}}\right) & +\bar{S}_{x 1}\left\{\frac{M c}{x^{2}} \bar{S}_{x 1}\left(\frac{M c}{x^{2}} a_{1}^{x} x^{m_{1}}\right)\right\} \\
+ & \bar{S}_{x 1}\left[\frac{M c}{x^{2}} \bar{S}_{x 1}\left\{\frac{M c}{x^{2}} \bar{S}_{x 1}\left(\frac{M c}{x^{2}} a_{1}^{x} x^{m_{1}}\right)\right\}\right]+\cdots
\end{aligned}
$$

On account of inequality (31) it is clear that the convergence of (39) carries with it the convergence of (38); this is at once apparent from a term by term comparison of the two series, since (39) is obtained from (38) by replacing each denominator by one at least as great. But (39) (except for its first term) is of the same form as the series in the first members of (15) and (19). Hence, just as in the case of the latter series, one proves that throughout the region $D_{1}$ the successive terms of (38) represent analytic functions and finally that the series itself represents an analytic function.

We shall now find an upper bound for the values of the $n-1$ functions $f_{i 1}(x), i=2, \cdots, n$, as $x$ varies within $P_{1}$; and, for similar variation of $x$, both upper and lower bounds for the values of $f_{11}(x)$. We have evidently

$$
\begin{aligned}
f_{i 1}(x)<\bar{S}_{x 1}\left(\frac{M c}{x^{2}} a_{1}^{x} x^{m_{1}}\right) & +\bar{S}_{x 1}\left\{\frac{M c}{x^{2}} \bar{S}_{x 1}\left(\frac{M c}{x^{2}} a_{1}^{x} x^{m_{1}}\right)\right\}+\cdots \\
& <\left|a_{1}^{x} x^{m_{1}}\right|\left\{\frac{M}{u-1}+\frac{M^{2}}{(u-1)^{2}}+\cdots\right\} \quad(i=2, \cdots, n),
\end{aligned}
$$

the latter inequality being deduced by aid of (15). Hence it is clear that

$$
\left|f_{i 1}(x)\right|<\left|a_{1}^{x} x^{m_{1}}\right| \frac{M}{u-M-1} \quad(i=2, \cdots, n) .
$$

Similarly, one has

$$
\left|f_{11}(x)\right|<\left|a_{1}^{x} x^{m_{1}}\right|\left(1+\frac{M}{u-M-1}\right)=\left|a_{1}^{x} x^{m_{1}}\right| \frac{u-1}{u-M-1} ;
$$


and also,

$$
\left|f_{11}(x)\right|>\left|a_{1}^{x} x^{m_{1}}\right|\left(1-\frac{M}{u-M-1}\right)=\left|a_{1}^{x} x^{m_{1}}\right| \frac{u-2 M-1}{u-M-1} .
$$

Combining these two inequalities we get

$$
\left|a_{1}^{x} x^{m_{1}}\right| \frac{u-2 M-1}{u-M-1}<\left|f_{11}(x)\right|<\left|a_{1}^{x} x^{m_{1}}\right| \frac{u-1}{u-M-1} .
$$

The desired results for the region $P_{1}$ are contained in inequalities (40) and (41).

Starting from (19) and working in a similar manner one obtains the following inequalities valid in $Q_{1}$ :

$$
\begin{gathered}
\left|f_{i 1}(x)\right|<\left|a_{1}^{x} x^{m_{1}}\right| \frac{A}{v-A} \quad(i=2, \cdots, n) ; \\
\left|a_{1}^{x} x^{m_{1}}\right| \frac{v-2 A}{v-A}<\left|f_{11}(x)\right|<\left|a_{1}^{x} x^{m_{1}}\right| \frac{v}{v-A} .
\end{gathered}
$$

By means of (27) we are able to determine the nature of $f_{i 1}(x), i=1, \ldots, n$, in the region $E_{1}$; for we have

$$
f_{i 1}(x-1)=\sum_{j=1}^{n} \bar{\psi}_{i j}(x-1) f_{j 1}(x) .
$$

Now the functions $f_{i 1}(x)$ are analytic throughout $P_{1}$. This solution may therefore be extended across the plane to the left by means of (44); it is evident from the nature of the process that the singularities of $f_{i 1}(x)$ for $x$ in $E_{1}$ are at the singularities of $\bar{\psi}_{i j}(x), i, j=1, \ldots, n$, and the points congruent to them on the left. The nature of the singularities which the functions $f_{i 1}(x)$ may have depends upon the particular functions $\bar{\psi}_{i j}(x)$ entering into a given particular system of equations. In special cases it may happen that some or all of the functions have no singularities at the points indicated.

Inequalities bounding the increase or decrease of $\left|f_{i 1}(x)\right|$ for $x$ in $E_{1}$ are not as complete as those for $x$ in $P_{1}$ and $Q_{1}$. We shall however obtain bounds for $\left|f_{i 1}(x)\right|$ when $x$ is in $E_{1 e}, E_{1 e}$ being defined as follows: Let $[S]$ be the set of points composed of all the singularities of $\bar{\psi}_{i j}(x), i, j=1, \ldots, n$, and the points congruent to them on the left. Enclose each point of $[S]$ by a circle of radius $\epsilon$ having the point for center. The part of $E_{1}$ no point of which is interior to one of these circles is the region $E_{1 \epsilon}$.

Referring to the definition of $\bar{\psi}_{i j}(x)$ in equation (28), it is easy to see that

$$
\underset{x=\infty}{L} \alpha_{i j}(x)=\left\{\begin{array}{l}
0 \text { if } j \neq i \\
a_{i}^{-1} \prod_{s=1}^{n} a_{x} \text { if } j=i
\end{array}\right.
$$


and that

Hence we have

$$
\underset{x=\infty}{L} \Delta=\prod_{s=1}^{n} a_{s}
$$

$$
\underset{x=\infty}{L} \bar{\psi}_{i j}(x)=\left\{\begin{array}{l}
0 \text { if } j \neq i, \\
a_{i}^{-1} \text { if } j=i .
\end{array}\right.
$$

From this it follows that for every positive constant $a$ such that $a<\left|a_{1}\right|$ there exists a positive constant $r$ for which

$$
\sum_{j=1}^{n}\left|\bar{\psi}_{i j}(x)\right|<a^{-1} \quad(i=1, \ldots, n) \quad|x| \geqq r .
$$

It will be assumed that $r$ is so taken that the circle $|x|=r$ cuts the boundary of $E_{1}$ in two points on the left of the origin and in no other point.

Since each of the functions $f_{i 1}(x)$ is analytic throughout $E_{1 \epsilon}$ it follows that a constant $\bar{N}$ exists such that

$$
\left|f_{i 1}\left(x^{\prime \prime}\right)\right|<\bar{N} \text { for } x^{\prime \prime} \text { in } E_{1 \mathrm{e}} \text { and } r \leqq\left|x^{\prime \prime}\right| \leqq r+1 .
$$

But from (27) we have

$f_{i 1}\left(x^{\prime \prime}-\mu\right)=\sum_{j_{1}=1}^{n} \bar{\psi}_{i j_{1}}\left(x^{\prime \prime}-\mu\right) \sum_{j_{2}=1}^{n} \bar{\psi}_{j_{1} j_{2}}\left(x^{\prime \prime}-\mu+1\right) \cdots \sum_{j_{\mu}=1}^{n} \bar{\psi}_{j_{\mu-1 j_{\mu}}}\left(x^{\prime \prime}-1\right) f_{j_{\mu} 1}\left(x^{\prime \prime}\right)$.

Hence we get

$$
\left|f_{i 1}\left(x^{\prime \prime}-\mu\right)\right|<\bar{N} a^{-\mu} \text {. }
$$

As $\mu$ varies over the range $\mu=0,1,2, \ldots, x^{\prime \prime}-\mu$ runs over all points in $E_{1 \mathrm{e}}$ and outside of the circle $|x|=r$. Also, $\left|f_{i 1}(x)\right|$ is finite in the part of $E_{1 \mathrm{e}}$ which is in the circle $|x|=r$. Hence it is clear that there exists a constant $N$, such that

$$
\left|f_{i 1}(x)\right|<N\left|a^{x}\right| \quad(i=1, \cdots, n) \quad x \text { in } E_{1 \epsilon} .
$$

The principal results of the section may be stated as follows :

The system of difference equations

$f_{i}(x+1)=\sum_{j=1}^{n} \psi_{i j}(x) f_{j}(x) \quad$ or $f_{i}(x-1)=\sum_{j=1}^{n} \bar{\psi}_{i j}(x-1) f_{j}(x)(i=1, \cdots, n)$, in which $\psi_{i j}(x)$ is single-valued and

$$
\psi_{i j}(x)=\delta_{i j} a_{i}\left(1+\frac{1}{x}\right)^{m_{i}}+\phi_{i j}(x), \quad \phi_{i j}(x)=\phi_{i j}^{\prime \prime} x^{-2}+\phi_{i j}^{\prime \prime \prime} x^{-3}+\cdots
$$

for $|x| \geqq R>1$,

$$
\delta_{i j}=0 \text { when } i \neq j ; \quad o_{i i}=1 \text {, }
$$

has a particular solution, $f_{i 1}(x)$, each function of which is analytic throughout the finite plane except at the singularities $[\alpha]$ of $\bar{\psi}_{i j}(x)$ and the points con- 
gruent to them on the left. If $D_{1}$ is defined by relations (30) to (34), then throughout any closed region in $D_{1}$ this solution is representable as a uniformly converging series in the form

$$
\begin{aligned}
f_{i 1}(x)=\delta_{i 1} a_{1}^{x} x^{m_{1}} & +S_{x i}\left(\phi_{i 1} a_{1}^{x} x^{m_{1}}\right)+S_{x i}\left\{\sum_{j=1}^{n} \phi_{i j} S_{x j}\left(\phi_{j 1} a_{1}^{x} x^{m_{1}}\right)\right\} \\
+ & S_{x i}\left[\sum_{j=1}^{n} \phi_{i j} S_{x j}\left\{\sum_{k=1}^{n} \phi_{j k} S_{x k}\left(\phi_{k 1} a_{1}^{x} x^{m_{1}}\right)\right\}\right]+\cdots \quad(i=1, \cdots, n) .
\end{aligned}
$$

The increase or decrease of these functions for $x$ in the corresponding regions $P_{1}$ and $Q_{1}$ is bounded as in the inequalities

$$
\begin{gathered}
\left|a_{1}^{x} x^{m_{1}}\right| \frac{u-2 M-1}{u-M-1}<\left|f_{11}(x)\right|<\left|a_{1}^{x} x^{m_{1}}\right| \frac{u-1}{u-M-1}, \text { for } x \text { in } P_{1} \\
\left|a_{1}^{x} x^{m_{1}}\right| \frac{v-A}{v-2 A}<\left|f_{11}(x)\right|<\left|a_{1}^{x} x^{m_{1}}\right| \frac{v}{v-A}, \text { for } x \text { in } Q_{1} \\
\left|f_{i 1}(x)\right|<\left|a_{1}^{x} x^{m_{1}}\right| \frac{M}{u-M-1} \quad(i=2, \ldots, n), \text { for } \dot{x} \text { in } P_{1} \\
\left|f_{i 1}(x)\right|<\left|a_{1}^{x} x^{m_{1}}\right| \frac{A}{v-A} \quad(i=2, \ldots, n), \text { for } x \text { in } Q_{1} .
\end{gathered}
$$

Moreover for any positive constant a such that $a<\left|a_{1}\right|$ there exists another corstant $N$ such that

$$
\left|f_{i 1}(x)\right|<N\left|a^{x}\right| \quad(i=1, \ldots, n), \text { for } x \text { in } E_{1 \mathrm{e}} .
$$

REMaRK. It is important for later use to point out the fact that, in so far as any statement in the conclusion of the theorem refers to the region $D_{1}$ or any part of it, it is independent of the nature of $\phi_{i j}(x), i, j=1, \cdots, n$, in the region exterior to $D_{1}$. Hence, if the functions $\phi_{i j}(x)$ are analytic in some $D$-region and satisfy the inequalities

$$
\left|\phi_{i j}(x)\right|<\frac{M c}{n|x|^{2}} \quad(i, j=1, \cdots, n), \text { for } x \text { in } D,
$$

then there exists a region $D_{1}$ having properties as in the theorem, and the system of equations has a particular solution $f_{i 1}(x)$ having in $D_{1}$ the properties of $f_{i 1}(x)$ of the theorem.

\section{§3. Existence of $n$ independent solutions of the normal system.}

For the system of equations considered in the preceding section we shall now obtain $n$ particular solutions which are independent and therefore constitute a fundamental system of solutions. 
Since by (37) it is seen that $f_{11}(x)$ is not identically zero, we may make the substitution

of which the inverse is

$$
\begin{gathered}
g_{1}(x)=f_{1}(x), \\
g_{i}(x)=f_{i}(x)-\frac{f_{i 1}(x)}{f_{11}(x)} f_{1}(x) \quad(i=2, \cdots, n),
\end{gathered}
$$

$$
f_{1}(x)=g_{1}(x), \quad f_{i}(x)=\frac{f_{i 1}}{f_{11}} \frac{(x)}{(x)} g_{1}(x)+g_{i}(x) \quad(i=2, \cdots, n),
$$

where $f_{i 1}(x), i=1, \cdots, n$, is the solution of $(24)$ obtained in the preceding section. Then we have

$$
\begin{aligned}
g_{i}(x+1)=f_{i}(x+1) & -\frac{f_{i 1}(x+1)}{f_{11}(x+1)} f_{1}(x+1) \\
= & \sum_{j=1}^{n}\left\{\psi_{i j}(x)-\frac{f_{i 1}(x+1)}{f_{11}(x+1)} \psi_{1 j}(x)\right\} f_{j}(x)
\end{aligned} \quad(i=2, \cdots, n) .
$$

Substituting in this equation the value of $f_{j}(x)$ obtained from (48) we have

$$
\begin{aligned}
& g_{i}(x+1)=\sum_{j=2}^{n}\left\{\psi_{i j}(x)-\frac{f_{i 1}(x+1)}{f_{11}(x+1)} \psi_{1 j}(x)\right\} g_{j}(x) \\
& +f_{1}(x) \sum_{j=1}^{n}\left\{\psi_{i j}(x)-\frac{f_{i 1}(x+1)}{f_{11}(x+1)} \psi_{1 j}(x)\right\}{\frac{f_{j 1}(x)}{f_{11}(x)}}^{(i=2, \cdots n) .}
\end{aligned}
$$

The last line of the expression may be written in the form

$$
\frac{g_{1}(x)}{f_{11}(x)}\left\{\sum_{j=1}^{n} \psi_{i j}(x) f_{j 1}(x)-\frac{f_{i 1}(x+1)}{f_{11}(x+1)} \sum_{j=1}^{n} \psi_{1 j}(x) f_{j 1}(x)\right\} .
$$

Since $f_{j 1}(x), j=1, \cdots, n$, is a solution of $(24)$, this reduces to

$$
\frac{g_{1}(x)}{f_{11}(x)}\left\{f_{i 1}(x+1)-\frac{f_{i 1}(x+1)}{f_{11}(x+1)} f_{11}(x+1)\right\},
$$

an expression which is identically zero. Hence in writing (49) the last line may be omitted. Thus we have

$$
g_{i}(x+1)=\sum_{j=2}^{n}\left\{\psi_{i j}(x)-\frac{f_{i 1}(x+1)}{f_{11}(x+1)} \psi_{1 j}(x)\right\} g_{j}(x) \quad(i=2, \cdots, n) .
$$

Substituting the values of $f_{i}(x), i=1, \ldots, n$, obtained from (48) in the first equation of system (24), we have

$$
g_{1}(x+1)=\sum_{j=1}^{n} \frac{f_{j 1}(x)}{f_{11}(x)} \psi_{1 j}(x) g_{1}(x)+\sum_{j=2}^{n} \psi_{1 j}(x) g_{j}(x) .
$$

Replacing in (51) and (50) the functions $\psi_{i j}(x)$ by their original values, we 
obtain the transformed system of equations :

$$
\begin{aligned}
& g_{1}(x+1)-a_{1}\left(1+\frac{1}{x}\right)^{m_{1}} g_{1}(x)=\sum_{j=1}^{n} \frac{f_{j 1}(x)}{f_{11}(x)} \phi_{1 j}(x) g_{1}(x)+\sum_{j=2}^{n} \phi_{1 j}(x) g_{j}(x) \\
& g_{i}(x+1)-a_{i}\left(1+\frac{1}{x}\right)^{m_{i}} g_{i}(x)=\sum_{j=2}^{n}\left\{\phi_{i j}(x)-\frac{f_{i 1}(x+1)}{f_{11}(x+1)} \phi_{1 j}(x)\right\} g_{j}(x)
\end{aligned}
$$

This system has the simple solution

$$
(i=2, \cdots, n) \text {. }
$$

$$
g_{1}(x)=f_{11}(x), \quad g_{i}(x)=0 \quad(i=2, \cdots, n) .
$$

By substitution of this solution in (48) one reproduces of course the known solution of (2t). We shall prove next that system (52), (53) has a second solution, and by means of it find a second solution of (24).

It will be noticed that the $n-1$ equations (53) involve only the $n-1$ functions $g_{2}(x), i=2, \ldots, n$; and therefore (53) is a system of $n-1$ equations in these unknowns. It satisfies the hypotheses of the theorem indicated in the remark at the close of $\S 2$; that is, there exist a constant $M^{\prime}(\geqq M)$ and a $D$. region such that each of the coefficient functions

$$
\phi_{i j}(x)-\frac{f_{i 1}(x+1)}{f_{11}(x+1)} \phi_{1 j}(x) \quad(i, j=2, \cdots, n),
$$

is analytic in $D$ and the inequality

$$
\left|\phi_{i j}(x)-\frac{f_{i 1}(x+1)}{f_{11}(x+1)} \phi_{1 j}(x)\right|<\frac{M^{\prime} c}{(n-1)|x|^{2}}
$$

is satisfied by every $x$ in $D$. The statement referring to the analytic nature of the coefficients is evidently true; for in a suitable $D$-region $f_{11}(x)$ does not vanish. It is clear also that there exists a constant $M^{\prime}$ such that the above inequality is true provided that there is a $D$-region in which $f_{i 1}(x+1) / f_{11}(x+1)$ is in absolute value equal to or less than 1. 'That this is true follows at once from inequalities (40) to (43).

From the above considerations and the theorem indicated in the remark referred to may be drawn several conclusions which are important for the succeeding argument :

1) There exist particular regions $P_{2}, Q_{2}, D_{2}$ and constants $c$ and $M_{1}\left(\geqq M^{\prime}\right)$ such that inequalities analogous to $(30)$ to $(34)$ hold and such that also

$$
u>2 M_{1}+1, \quad A_{1} / v<\frac{1}{2}, \quad\left|f_{i 1}(x) / f_{11}(x)\right| \leqq 1, \text { for } x \text { in } D_{2} .
$$

2) The system of equations (53) has a particular solution, $g_{i 2}(x), i=2, \ldots, n$, each function of which is analytic throughout $D_{2}$.

3) The increase or decrease of the functions which constitute this solution is 
bounded as follows :

$$
\begin{aligned}
& \left|a_{2}^{x} x^{m_{2}}\right| \frac{v-2 A_{1}}{v-A_{1}}<\left|g_{22}(x)\right|<\left|a_{2}^{x} x^{m_{2}}\right| \frac{v}{v-A_{1}}, \\
& \left|g_{i 2}(x)\right|<\left|a_{2}^{x} x^{m_{2}}\right| \frac{A_{1}}{v-A_{1}} \quad(i=3, \cdots, n), \\
& \left|a_{2}^{x} x^{m_{2}}\right| \frac{u-2 M_{1}-1}{u-M_{1}-1}<\left|g_{22}(x)\right|<\left|a_{2}^{x} x^{m_{2}}\right| \frac{u-1}{u-M_{1}-1}, \text { for } x \text { in } Q_{2} ; \\
& \left|g_{i 2}(x)\right|<\left|a_{2}^{x} x^{m_{2}}\right| \frac{M_{1}}{u-M_{1}-1} \quad(i=3, \ldots, n),
\end{aligned}
$$

These $n-1$ functions are a part of a particular solution of the system (52), (53). To find the other function $g_{12}(x)$ of this solution we have the non-homogeneous equation

$$
\begin{aligned}
g_{12}(x+1)-a_{1}\left(1+\frac{1}{x}\right)^{m_{1}} & g_{12}(x) \\
& =\sum_{j=1}^{n} \frac{f_{j 1}(x)}{f_{11}(x)} \phi_{1 j}(x) g_{12}(x)+\sum_{j=2}^{n} \phi_{1 j}(x) g_{j 2}(x)
\end{aligned}
$$

We obtain first a solution of the reduced equation

$$
g(x+1)-a_{1}\left(1+\frac{1}{x}\right)^{m_{1}} g(x)=\sum_{j=1}^{n} \frac{f_{j 1}(x)}{f_{11}(x)} \phi_{1 j}(x) g(x),
$$

an equation which satisfies the hypotheses of the theorem indicated in the remark at the close of $\S 1$, as is shown by relations (54) and those indicated in the lines preceding (54). Accordingly it has a particular solution $\bar{g}(x)$ analytic and not zero throughout $D_{2}$ and approximately characterized by the inequalities

$$
\left|a_{1}^{x} x^{m_{1}}\right| \frac{v-2 A_{1}}{v-A_{1}}<|\bar{g}(x)|<\left|a_{1}^{x} x^{m_{1}}\right| \frac{v}{v-A_{1}}, \text { for } x \text { in } Q_{2} ;
$$

$$
\left|a_{1}^{x} x^{m_{1}}\right| \frac{u-2 M_{1}-1}{u-M_{1}-1}<|\bar{g}(x)|<\left|a_{1}^{x} x^{m_{1}}\right| \frac{u-1}{u-M_{1}-1}, \text { for } x \text { in } P_{2} .
$$

Substituting in (57)

$$
g_{12}(x)=h(x) \bar{g}(x)
$$

and making use of the fact that $\bar{g}(x)$ satisfies (58), one obtains by easy reduction

$$
h(x+1)-h(x)=\sum_{j=2}^{n} \frac{\phi_{1 j}(x) g_{j 2}(x)}{\bar{g}(x+1)}=\eta(x) .
$$

It is clear that $\eta(x)$ is analytic throughout $D_{2}$.

Bounds of increase or decreas? of $\eta(x)$ may be readily obtained. From (55), 
(56), and (59) we have

$$
\begin{gathered}
|\eta(x)|<\left|\left(\frac{a_{2}}{a_{1}}\right)^{x} x^{m_{2}-m_{1}-2}\right| \frac{M v}{v-2 A_{1}}, \text { for } x \text { in } Q_{2} ; \\
|\eta(x)|<\left|\left(\frac{a_{2}}{a_{1}}\right)^{x} x^{m_{2}-m_{1}-2}\right| \frac{M(u-1)}{u-2 M_{1}-1}, \text { for } x \text { in } P_{2} .
\end{gathered}
$$

From the first of these inequalities it is clear that the series

$$
s(x)=\eta(x-1)+\eta(x-2)+\eta(x-3)+\cdots
$$

is uniformly convergent throughout any closed region in $Q_{2}$; and hence the function $s(x)$ is analytic in $Q_{2}$. We have also

$$
s(x+1)-s(x)=\eta(x),
$$

so that $h(x)=s(x)$ is a solution of (61) which is analytic in $Q_{2}$. But its behavior near the positive real axis is complicated; and the solution of (52), (53), and hence of (24), to which it leads is composed of functions which are complicated in their character in the same region. 'We seek therefore another solution, one which will avoid this difficulty near the real axis; it will of course differ from $s(x)$ by a periodic function.

A solution of (61) in the form of a contour integral, the path of integration going to infinity along two parallel lines, will be found to serve our purpose. We form the integral

$$
I(x)=\int_{L} \frac{\eta(z) d z}{1-e^{2 \pi i(x-z)}},
$$

where the path of integration $L$ is yet to be chosen. Since $\eta(x)$ is analytic throughout $D_{2}$, it is evident that all the infinities which the integrand has in $D_{2}$ are at the points $x \pm r$, where $r$ is zero or a positive integer. Let $A B$ be the straight line containing all these points. Let the path of integration $L$ or $C K R H$ lie entirely in $D_{2}$ and be formed in the following manner: It consists

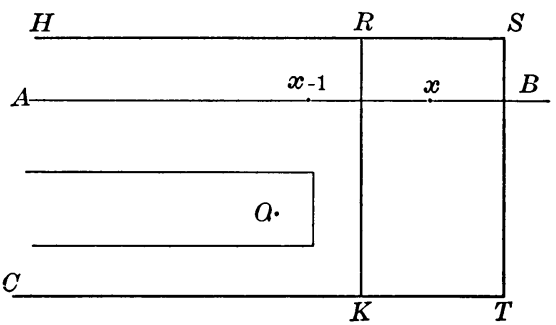

FIG. 2.

of three parts : a part $R H$ lying entirely above $A B$ and extending to infinity in a negative direction parallel to the axis of reals; a part $K C$ lying below $A B$ and 
extending to infinity in a negative direction parallel to the axis of reals; a finite part $K R$ which crosses $A B$ once between $x-1$ and $x$ and at no other point. Moreover the path $C K R H$ is such that $E_{2}$ lies entirely to the left of it. From (62) it is evident that the integral along such a path has a meaning. It is clear that for a particular $x$ the path of integration may be deformed at will without changing the value of $I(x)$, provided that during the deformation it has always the properties stated in the definition of $C K R H$. Furthermore, $I(x)$ is analytic in $D_{2}$.

We show that $h(x)=I(x)$ is a solution of $(61)$. In view of the fact that the integrand considered as a function of $x$ is periodic of period 1 it is clear that we have

$$
I(x+1)=\int_{L^{\prime}} \frac{\eta(z) d z}{1-e^{2 \pi} \overline{i(x-z)}},
$$

where $L^{\prime}$ is the path $C T S H$. Now

$$
\int_{L^{\prime}}-\int_{L}=\int_{K T S R K}
$$

But by Cauchy's theorem the last integral is equal to $\eta(x)$. Hence, subtracting (65) from (66), member by member, we have

that is,

$$
I(x+1)-I(x)=\eta(x)
$$

$$
h(x)=I(x)
$$

is a solution of (61). It is this particular solution which we shall take for the value of $h(x)$ in the remaining discussion. It is analytic in $D_{2}$, as we have seen.

It is necessary, for later use, to know a bound to the increase or decrease of $|h(x)|$ for $x$ in $Q_{2}$ and $P_{2}$. We consider the two regions separately, first taking the region $Q_{2}$. On account of $(64)$ and the analytic nature of both $h(x)$ and $s(x)$ in $Q_{2}$, it is evident that $h(x)-s(x)$ is a periodic function of $x$ of period 1 and analytic throughout $Q_{2}$. Now

$$
|h(x)| \leqq|h(x)-s(x)|+|s(x)| ;
$$

accordingly we find upper bounds for $|h(x)-s(x)|$ and $|s(x)|$ separately. Consider the function $h(x)-s(x)$ for $x=u \pm v v \overline{-1}, x$ being in $Q_{2}$, where $v$ is fixed and where $u$ runs over any stretch of length 1 . For such variation of $x,|h(x)-s(x)|$ has evidently a maximum which is entirely independent of the range for $u$ but depends directly on $v$. Call this maximum $m( \pm v)$. Then we have

$$
|h(x)-s(x)| \leqq m( \pm v), \text { for } x \text { in } Q_{2},
$$

where $m( \pm v)$ is a single-valued function of $\pm v$. 
Now

$$
|s(x)| \leqq \sum_{i=1}^{\infty}|\eta(x-i)|<\frac{M v}{v-2 A_{1}} \sum_{i=1}^{\infty}\left|\left(\frac{a_{2}}{a_{1}}\right)^{x-i}(x-i)^{m_{2}-m_{1}-2}\right|,
$$

by (62). Hence we have

$$
|s(x)|<\frac{M v}{v-2 A_{1}}\left|\left(\frac{a_{2}}{a_{1}}\right)^{x} x^{m_{2}-m_{1}-2}\right| \sum_{i=1}^{\infty}\left|\left(\frac{a_{1}}{a_{2}}\right)^{i}\left(1-\frac{i}{x}\right)^{m_{2}-m_{1}-2}\right| .
$$

Therefore we obtain the result

$$
|s(x)|<\frac{B_{1}}{|x|^{2}}\left|\left(\frac{a_{2}}{a_{1}}\right)^{x} x^{m_{2}-m_{1}}\right|, \text { for } x \text { in } Q_{2},
$$

where $B_{1}$ is a properly chosen constant. Then, finally, from (67), (68), (69), we have

$$
|h(x)|<m( \pm v)+\frac{B_{1}}{|x|^{2}}\left|\left(\frac{a_{2}}{a_{1}}\right)^{x} x^{m_{2}-m_{1}}\right|, \text { for } x \text { in } Q_{2} .
$$

In order to find an upper bound for $|h(x)|$ when $x$ is in $P_{2}$ we proceed as follows: Let $A B$ be the line parallel to the axis of reals and containing the

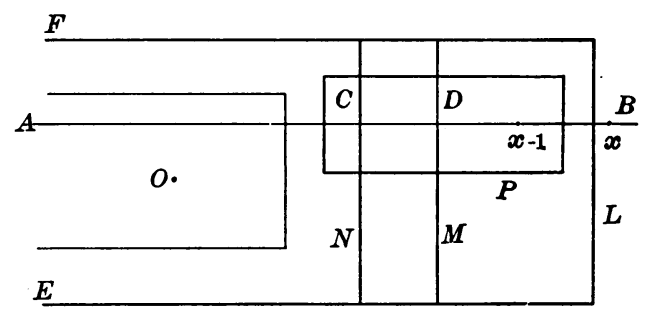

FIG. 3.

point $x$. Construct the contours $E L F, E M F, E N F, P$ as in the figure, where the stretch $C D$ is equal to $\frac{1}{2}$. Let us denote by $I_{L}(x), I_{M}(x), I_{N}(x)$, $I_{P}(x)$ respectively the integral

$$
\int \frac{\eta(z) d z}{1-e^{2 \pi i(x-z)}}
$$

taken around these contours. For any value of $x$, either $I_{M}(x)$ or $I_{N}(x)$ represents an integral which is convergent, though the path of integration of one of them may pass through an infinity of the integrand. $I_{L}(x)$ and $I_{P}(x)$ always have a meaning, if it is understood that the contour $P$. may vary so as not to. cross $A B$ at a point congruent to $x$.

Since $h(x) \equiv I_{L}(x)$, we clearly have

$$
\begin{aligned}
& |h(x)| \leqq\left|I_{M}(x)\right|+\bar{I}_{P}(x), \\
& |h(x)| \leqq\left|I_{N}(x)\right|+\bar{I}_{P}(x),
\end{aligned}
$$


where $\bar{I}_{P}(x)$ is the quantity obtained by applying to $I_{P}(x)$ the calculus of residues and replacing by its absolute value each term of the sum so obtained. Let $x$ vary along $A B$. For each $x$ take the smaller of the quantities $\left|I_{M}(x)\right|$ and $\left|I_{N}(x)\right|$; call this quantity $\tau(x)$. From the nature of the integrand in $I(x)$, it is evident that $\tau(x)$ has a maximum; we denote this maximum by $\mu( \pm v)$, since it depends on $\pm v$ alone. Thus we obtain

$$
|h(x)| \leqq \mu( \pm v)+\bar{I}_{P}(x), \quad \text { for } x \text { in } P_{2} .
$$

Now from (62) and the calculus of residues it is clear that when $x$ is in $P_{2}$ we have

Hence we obtain

$$
\left|I_{P}(x)\right|<\frac{M(u-1)}{u-2 M_{1}-1} \sum_{i=1}^{\infty}\left|\left(\frac{a_{2}}{a_{1}}\right)^{x-i}(x-i)^{m_{2}-m_{1}-2}\right| \text {. }
$$

$$
\left|I_{P}(x)\right|<\frac{B_{2}}{|x|^{2}}\left|\left(\frac{a_{2}}{a_{1}}\right)^{x} x^{m_{2}-m_{1}}\right|, \quad \text { for } x \text { in } P_{2},
$$

where $B_{2}$ is a properly chosen constant. Therefore we have

$$
|h(x)|<\mu( \pm v)+\frac{B_{2}}{|x|^{2}}\left|\left(\frac{a_{2}}{a_{1}}\right)^{x} x^{m_{2}-m_{1}}\right|, \text { for } x \text { in } P_{2} .
$$

Since $h(x)$ and $\bar{g}(x)$ are known functions, both analytic throughout $D_{2}$, equation (60) gives us a function $g_{12}(x)$ analytic in $D_{2}$. From (59), (70), ( $(\overline{70})$ we have

$$
\begin{aligned}
& \left|g_{12}(x)\right|<\left|a_{1}^{x} x^{m_{1}}\right| \frac{v \cdot m( \pm v)}{v-A_{1}}+\left|a_{2}^{x} x^{m_{2}}\right| \frac{B_{2}}{|x|^{2}} \cdot \frac{v}{v-A_{1}}, \text { for } x \text { in } Q_{2} \\
& \left|g_{12}(x)\right|<\left|a_{1}^{x} x^{m_{1}}\right| \frac{\mu( \pm v)(u-1)}{u-M_{1}-1}+\left|a_{2}^{x} x^{m_{2}}\right| \frac{B_{2}}{|x|^{2}} \cdot \frac{u-1}{u-M_{1}-1}, \text { for } x \text { in } P_{2} .
\end{aligned}
$$

From (48) we now have a second solution of $(24)$; calling it $f_{i 2}(x), i=1, \ldots, n$, we may write

$$
\begin{aligned}
& f_{12}(x)=g_{12}(x) \\
& f_{i 2}(x)=\frac{f_{i 1}(x)}{f_{11}(x)} g_{12}(x)+g_{i 2}(x) \quad(i=2, \cdots, n) .
\end{aligned}
$$

Also from (55), (56), (71) we have the following inequalities :

$$
\left\{\begin{array}{l}
\left|f_{12}(x)\right|<\left|a_{2}^{x} x^{m_{2}}\right| \frac{B_{2}}{|x|^{2}} \cdot \frac{v}{v-A_{1}}+\left|a_{1}^{x} x^{m_{1}}\right| \frac{v \cdot m( \pm v)}{v-A_{1}}, \\
\left|f_{22}(x)\right|<\left|a_{2}^{x} x^{m_{2}}\right| \frac{v}{v-A_{1}}+\left|f_{12}(x)\right|, \\
\left|f_{22}(x)\right|>\left|a_{2}^{x} x^{m_{2}}\right| \frac{v-2 A_{1}}{v-A_{1}}-\left|f_{12}(x)\right|, \\
\left|f_{i 2}(x)\right|<\left|a_{2}^{x} x^{m_{2}}\right| \frac{A_{1}}{v-A_{1}}+\left|f_{12}(x)\right| \quad(i=3, \ldots, n),
\end{array} \text { for } x \text { in } Q_{2} ;\right.
$$




$$
\left\{\begin{array}{l}
\left|f_{12}(x)\right|<\left|a_{2}^{x} x^{m_{2}}\right| \frac{B_{2}}{|x|^{2}} \cdot \frac{u-1}{u-M_{1}-1}+\left|a_{1}^{x} x^{m_{1}}\right| \frac{\mu( \pm v)(u-1)}{u-M_{1}-1} \\
\left|f_{22}(x)\right|<\left|a_{2}^{x} x^{m_{2}}\right| \frac{u-1}{u-M_{1}-1}+\left|f_{12}(x)\right|, \\
\left|f_{22}(x)\right|>\left|a_{2}^{x} x^{m_{2}}\right| \frac{u-2 M_{1}-1}{u-M_{1}-1}-\left|f_{12}(x)\right| \\
\left|f_{i 2}(x)\right|<\left|a_{2}^{x} x^{m_{2}}\right| \frac{M_{1}}{u-M_{1}-1}+\left|f_{12}(x)\right| \quad(i=3, \ldots, n)
\end{array} \quad \text { for } x \text { in } P_{2} .\right.
$$

It should be observed that the results of this paragraph, inasmuch as they refer only to the region $D_{2}$, are again independent of the nature of the functions $\phi_{i j}(x)$ in $E_{2}$.

If $n>2$ we may obtain a third solution of (24) in the following manner: The solution of (24) just obtained depends intimately upon the two smallest $a$ 's in the system, as inequalities (73), (74) show. Hence it follows that (53) has a corresponding solution depending in like manner upon the two smallest $a$ 's in that system; this solution we shall call $g_{i 3}(x), i=2, \ldots, n$. It is analytic throughout some region $D_{3}$ and satisfies the inequalities corresponding to (73):

$$
\begin{aligned}
& \left|g_{23}(x)\right|<\left|a_{3}^{x} x^{m_{3}}\right| \frac{B_{3}}{|x|^{2}} \cdot \frac{v}{v-A_{2}}+\left|a_{2}^{x} x^{m_{2}}\right| \frac{v \cdot m_{1}( \pm v)}{v-A_{2}}, \\
& \left|g_{33}(x)\right|<\left|a_{3}^{x} x^{m_{3}}\right| \frac{v}{v-A_{2}}+\left|g_{23}(x)\right|, \\
& \left|g_{33}(x)\right|>\left|a_{3}^{x} x^{m_{3}}\right| \frac{v-2 A_{2}}{v-A_{2}}-\left|g_{23}(x)\right|, \quad \text { for } x \text { in } Q_{3} ; \\
& \left|g_{i 3}(x)\right|<\left|a_{3}^{x} x^{m_{3}}\right| \frac{A_{2}}{v-A_{2}}+\left|g_{23}(x)\right| \quad(i=4, \ldots, n),
\end{aligned}
$$

where $D_{3}, Q_{3}, m_{1}( \pm v), A_{2}, B_{3}$ are defined in a manner analogous respectively to the definitions of $D_{2}, Q_{2}, m( \pm v), A_{1}, B_{1}$. Also there exists of course a set of inequalities similar to (74) for $x$ in $P_{3}$; it is not necessary to write them out.

We have thus $n-1$ functions of a third solution of (52), (53). To find the other function, which we will call $g_{13}(x)$, we have an equation corresponding to (57) above. If we write

$$
g_{13}(x)=h_{1}(x) \bar{g}(x),
$$

where $\bar{g}(x)$ is defined as before, it is easy to see that $h_{1}(x)$ is a solution of

$$
h_{1}(x+1)-h_{1}(x)=\sum_{j=2}^{n} \frac{\phi_{1, j}(x) g_{j 3}(x)}{\bar{g}(x+1)} \equiv \eta_{1}(x) \text {. }
$$


From inequalities (59), (75) and those for $x$ in $P_{3}$ corresponding to (75) and the fact that $\eta_{1}(x)$ is analytic throughout $D_{3}$ it follows that the method used for solving (61) is applicable to (76) and that a solution may be obtained as before in the form of a contour integral. We thus find a third particular solution of (52), (53). Just as before this leads to a solution of (24). It is evident that this process may be continued until $n$ particular solutions of (24) are obtained and that there exists a $D$-region in which each of the $n^{2}$ functions of these solutions is analytic. We shall call these $n$ solutions

$$
f_{1 j}(x), f_{2 j}(x), \cdots, f_{n j}(x) \quad(j=1, \cdots, n) .
$$

It is clear that we may employ (27) to extend each of these solutions aoross the plane to the left and in this way prove that each function $f_{i j}(x)$ is analytic throughout the finite plane except at the singularities of $\bar{\psi}_{i j}(x)$ and the points congruent to them on the left.

A reference to the discussion (in the preceding section) of the bounds of $\left|f_{i 1}(x)\right|$ in the region $E_{1}$ will bring out the fact that an argument similar to that employed there is applicable to any one of the $n$ particular solutions which we have obtained, and that the result in each case takes the same form as that obtained for the first solution.

Let us now write

$$
f_{i j}(x)=a_{j}^{x} x^{m_{j}} U_{i j}(x) \quad(i, j=1, \cdots, n),
$$

where the functions $U_{i j}(x)$ are defined by these equations. Inequalities (40) to $(43),(73),(74)$ and the analogous inequalities for the other solutions enable us to conclude (without further argument) that when $x$ approaches infinity in the positive direction along any line parallel to the real axis we have*

$$
\lim U_{i j}(x)=\left\{\begin{array}{l}
0 \text { if } i \neq j, \\
1 \text { if } i=j .
\end{array}\right.
$$

This fact will enable us to prove the independence of the solutions (77). The necessary and sufficient condition for such independence $\dagger$ is that the determinant of which $f_{i j}(x)$ is the general element is not identically zero. Now we have

$$
\left|f_{i j}(x)\right|=a_{1}^{x} a_{2}^{x} \cdots a_{n}^{x} x^{m_{1}+m_{2}+\cdots+m_{n}}\left|U_{i j}(x)\right| \text {. }
$$

From this result and (78) it follows that the determinant of which $f_{i j}(x)$ is the

* This result brings into prominent notice the important question of the asymptotic character of the solutions for every approach to infinity. The full discussion of this subject requires a separate investigation. There are several excellent papers by HoRx, ForD, PerRoN and others treating the highly important special case in which $x$ runs over a set of points $x_{0}, x_{0}+1, x_{0}+2, \cdots$. See references on pp. 160, 190, 191 of HoRn's paper in Crelle's Journal, vol. 138 (1910). To the papers which he lists should be added one by Perron in Jahresbericht der deutschen Mathematiker-Vereinigung, vol. 19 (1910), pp. 129-137. See also the paper by W ATson referred to in the introduction to the present paper.

† Cf. Pincherle and Amaldi, Le operazione distributive, p. 218. 
general element is not identically zero. Hence the solutions (77) are independent and form therefore a fundamental system of solutions of the system (24); and hence the general solution is

$$
f_{i}(x)=\sum_{j=1}^{n} \omega_{j}(x) f_{i j}(x)
$$

$(i=1, \cdots, n)$,

where the functions $\omega_{j}(x), j=1, \cdots, n$, are arbitrary periodic functions of period 1.

In the theorem below, the inequalities bounding $\left|f_{i j}(x)\right|, i, j=1, \cdots, n$, are not given; otherwise the principal results of the section are as follows:

The system of equations

$f_{i}(x+1)=\sum_{j=1}^{n} \psi_{i j}(x) f_{j}(x)$ or $f_{i}(x-1)=\sum_{j=1}^{n} \bar{\psi}_{i j}(x-1) f_{j}(x) \quad(i=1, \cdots, n)$, $\psi_{i j}(x)$ being restricted as in the theorem of $\S 2$, has the fundamental system of solutions

$$
f_{1 j}(x), f_{2 j}(x), \cdots, f_{n j}(x) \quad(j=1, \cdots, n),
$$

determined in the preceding pages and having the following properties : Each function $f_{i j}(x)$ is analytic throughout the finite plane except at the singularities of $\bar{\psi}_{i j}(x)$ and the points congruent to them on the left. The general solution of the system of equations is

$$
f_{i}(x)=\sum_{j=1}^{n} \omega_{j}(x) f_{i j}(x) \quad(i=1, \cdots, n),
$$

where the functions $\omega_{j}(x), j=1, \ldots, n$, are arbitrary periodic functions of period 1. Further, for $x$ approaching infinity along any line parallel to the positive real axis we have

$$
\lim f_{i j}(x) a_{j}^{-x} x^{-m_{\jmath}}=\left\{\begin{array}{lll}
0 & \text { if } & i \neq j \\
1 & \text { if } & i=j
\end{array}\right.
$$

§4. Reduction to normal form. General existence theorems.

Let

$$
G_{i}(x+1)=x^{a} \sum_{j=1}^{n} a_{i j}(x) G_{j}(x) \quad(i=1, \cdots, n),
$$

be a system of $n$ first order linear homogeneous difference equations involving $n$ unknown functions $G_{1}(x), \ldots, G_{n}(x)$ of the complex variable $x$, the known quantities entering into the equation being defined as follows :

1) $\alpha$ is a constant ;

2) the functions $a_{i j}(x)$ are single-valued and

$$
a_{i j}(x)=a_{i j}+a_{i j}^{\prime} x^{-1}+a_{i j}^{\prime \prime} x^{-2}+\cdots \quad(i, j=1, \cdots, n) \quad|x|>R ;
$$


3) the constants $a_{i j}$ are such that the roots of the equation

$$
\left|a_{i j}-\delta_{i j} \rho\right|=0, \quad \delta_{i j}=\left\{\begin{array}{l}
0 \text { if } i \neq j, \\
1 \text { if } i=j,
\end{array}\right.
$$

are in absolute value different from each other and from zero.

Equation (80) is the characteristic equation associated with (79). For convenience we assume that its roots $a_{1}, \ldots, a_{n}$ are arranged so that

$$
0<\left|a_{1}\right|<\left|a_{2}\right|<\cdots<\left|a_{n}\right| \text {. }
$$

The object of this section is to extend the theorem of $\S 3$ to the system in its more general form and then to apply the result to a single equation of the $n$th order. The work is carried out by first reducing (79) to the normal form already studied. In this discussion two properties of the $\Gamma$-function will be assumed; namely, that it is analytic to the right of the axis of imaginaries, and that it satisfies the functional equation

$$
\Gamma(x+1)=x \Gamma(x) \text {. }
$$

It is obvious that $\alpha$ can be reduced to zero by means of the transformation

$$
G_{i}(x)=\{\Gamma(x)\}^{a} \bar{G}_{i}(x) .
$$

Effecting this substitution in (79) and reducing by means of (82) we have

$$
\bar{G}_{i}(x+1)=\sum_{j=1}^{n} a_{i j}(x) \bar{G}_{j}(x) \quad(i=1, \cdots, n) .
$$

The first step in the normalization of (84) will be made by means of a linear transformation with constant coefficients. This done, a further reduction (of a different type) will enable us to remove the term in $x^{-1}$ from the resulting: coefficient functions $\bar{a}_{i j}(x), i \neq j$.

Let us put

$$
\bar{G}_{i}(x)=\sum_{j=1}^{n} \alpha_{i j} F_{j}(x) \quad(i=1, \cdots, n),
$$

where the determinant $\left|\alpha_{i j}\right|$ is different from zero. Making this substitution in (84) and solving for $F_{i}(x+1)$ one may write the result in the form

$$
F_{i}(x+1)=\sum_{j=1}^{n} \bar{a}_{i j}(x) F_{j}(x) \quad(i=1, \cdots, n),
$$

where the functions $\bar{a}_{i j}(x)$, being linear combinations of the functions $a_{i j}(x)$ with constant coefficients, may be expanded in powers of $x^{-1}$ for $|x|>R^{\prime}$.

In connection with this transformation, let us consider the effect in the linear substitution

$$
y_{i}^{\prime}=\sum_{j=1}^{n} a_{i j} y_{j} \quad(i=1, \cdots, n)
$$


of replacing $y_{i}^{\prime}$ and $y_{i}$ by their values as given in the transformation

$$
y_{i}=\sum_{j=1}^{n} \alpha_{i j} \bar{y}_{j}, \quad y_{i}^{\prime}=\sum_{j=1}^{n} \alpha_{i j} \bar{y}_{j}^{\prime} \quad(i=1, \cdots, n) .
$$

Having made these substitutions, solve for $\bar{y}_{i}^{\prime}$ and write the result

$$
\bar{y}_{i}^{\prime}=\sum_{j=1}^{n} \bar{a}_{i j} \bar{y}_{j} \quad(i=1, \cdots, n) .
$$

Comparing the operations of this paragraph with those of the preceding, it is easy to see that the constant term in the function $\bar{a}_{i j}(x)$ must be identical with $\bar{a}_{i j}$ for each pair of values $i$ and $j$. But, on account of (81), it follows from the well-known theory* of linear substitutions that a proper determination of $\alpha_{i j}$ in (88) will transform (87) to the simpler system

$$
\bar{y}_{i}^{\prime}=a_{i} \bar{y}_{i} \quad(i=1, \cdots, n) .
$$

Suitable values of $\alpha_{i j}$ may be determined $*$ by solving the following $n$ systems. of equations :

$$
\sum_{j=1}^{n} a_{i j} \alpha_{j \tau}-a_{\tau} \alpha_{i \tau}=0 \quad(i, \tau=1, \cdots, n),
$$

a separate system being formed for each $\tau$. That each system (90) is consistent follows from the fact that $\rho=a_{\tau}$ is a solution of the characteristic equation (80). Hence the ratios of $\alpha_{i \tau}$ for fixed $\tau$ and $i=1, \ldots, n$ are determined by (90). Out of the infinity of proportional solutions of each of these $n$ systems of equations we suppose a particular one chosen, and that the quantities $\alpha_{i j}$ have these fixed values throughout the remaining discussion.

If the values of $\alpha_{i j}$ so determined are used in (85), that transformation throws (84) into the simpler system

$$
F_{i}(x+1)=a_{i} F_{i}(x)+\sum_{j=1}^{n} b_{i j}(x) F_{j}(x) \quad(i=1, \cdots, n),
$$

where

$$
b_{i j}(x)=b_{i j} x^{-1}+b_{i j}^{\prime} x^{-2}+\cdots \quad(i, j=1, \cdots, n) \quad|x|>R^{\prime} .
$$

In order to remove the term in $x^{-1}$ from each of the coefficient functions. $b_{i j}(x), i \neq j$, we employ a transformation of the form

$$
F_{i}(x)=f_{i}(x)+\sum_{j=1}^{n} \frac{\beta_{i j}}{x} f_{j}(x), \quad \beta_{i i}=0, \quad(i=1, \cdots, n),
$$

where the constants $\beta_{i_{j}}, i \neq j$, are as yet undetermined. The determinant of this transformation is

$$
\Delta \equiv\left|\delta_{i j}+\frac{\beta_{i j}}{x}\right|=1+\text { terms in } x^{-2}, x^{-3}, \ldots
$$

* Cf. HoRn, Geubhnliche Differentialyleichungen, pp. 72-74. 
Substituting these values for $F_{i}(x)$ in (91) and writing only those terms which do not involve $x^{-2}, x^{-3}, \ldots$, we have, when $|x|>R$,

$$
\begin{array}{r}
f_{i}(x+1)+\sum_{j=1}^{n}\left(\frac{\beta_{i j}}{x+1}\right) f_{j}(x+1)=a_{i,} f_{i}(x)+a_{i} \sum_{j=1}^{n} \frac{\beta_{i j}}{x} f_{j}(x) \\
\quad+\sum_{j=1}^{n}\left\{\left(\frac{b_{i j}}{x}+\cdots\right)\left[f_{j}(x)+\sum_{k=1}^{n} \frac{\beta_{i k}}{x} f_{k}(x)\right]\right\} \\
=\left(a_{i}+\frac{b_{i i}}{x}+\cdots\right) f_{i}(x)+\sum_{j=1}^{n}\left(\frac{a_{i} \beta_{i j}+b_{i j}}{x}+\cdots\right) f_{j}(x),
\end{array}
$$

the accent in $\Sigma^{\prime}$ denoting that in the summation the term for which $j=i$ is to be omitted.

It is necessary to solve (93) for $f_{i}(x+1)$. We proceed thus : The cofactor $\Delta_{i j}$ of the element in the $i$ th row and $j$ th column of $\Delta$ has clearly no constant term, except when $i$ equals $j$, when there is a constant term $1:$ the term in $1 / x$ for $i \neq j$ is clearly $-\beta_{j i} / x$. For, if we cross out the $i$ th row and $j$ th column of $\Delta$, there remain $n-2$ elements with a constant term 1 ; and therefore the expansion of this minor can contain only one term in $1 / x$, namely that term which contains all the $n-2$ factors 1 and whose remaining factor is not in the same rows or columns as these. Therefore this term is $\pm \beta_{j i} / x$; and, since an even number of interchanges of rows and columns bring the elements $\beta_{i j} / x$ and $\beta_{j i} / x$ to the place in the first row second column and second row first column respectively, the negative sign must be chosen. In the cofactor $\Delta_{i i}$ there is no term in $1 / x$; for every term in the expansion containing one factor $1 / x$ necessarily contains another. Hence the cofactors may be written

$$
\begin{gathered}
\Delta_{i j}=-\frac{\beta_{j i}}{x}+\text { terms in } x^{-2}, x^{-3}, \ldots, \quad(\imath \neq j) ; \\
\Delta_{i i}=1+\text { terms in } x^{-2}, x^{-3}, \ldots
\end{gathered}
$$

Solving (93) for $f_{i}(x+1)$ we readily obtain

$$
\begin{array}{r}
(94) \bar{\Delta} f_{i}(x+1)=\left(a_{i}+\frac{b_{i i}}{x}+\cdots\right) f_{i}(x)+\sum_{j=1}^{n}\left\{\frac{\left(a_{i}-a_{j}\right) \beta_{i j}+b_{i j}}{x}+\cdots\right\} f_{j}(x) \\
(i=1, \cdots, n),
\end{array}
$$

$\bar{\Delta}$ denoting the determinant

$$
\bar{\Delta}=\left|\delta_{1 j}+\frac{\beta_{i j}}{x+1}\right| .
$$

For values of $x$ such that $|x| \geqq R^{\prime \prime}, R^{\prime \prime}$ being a properly chosen constant greater than $R^{\prime}$ and greater than 1 , the determinant $\bar{\Delta}$ is everywhere different from zero and is expansible in the form $1+$ terms in $x^{-2}, x^{-3}, \ldots$ If we divide by 
$\bar{\Delta}$ we obtain therefore

$$
\begin{array}{r}
f_{i}(x+1)=\left(a_{i}+\frac{b_{i i}}{x}+\cdots\right) f_{i}(x)+\sum_{j=1}^{n}\left\{\frac{\left(a_{i}-a_{j}\right) \beta_{i j}+b_{i j}}{x}+\cdots\right\} f_{j}(x) \\
|x| \geqq R^{\prime \prime}
\end{array}
$$

the terms of order $x^{-2}, x^{-3}, \ldots$ in the coefficients of $f_{j}(x)$ in the right hand member are of course different from the corresponding terms in (94).

On account of $(81)$ it is always possible to determine $\beta_{i j}, i \neq j$, so that

$$
\left(a_{i}-a_{j}\right) \beta_{i j}+b_{i j}=0 \text {. }
$$

When the values of $\beta_{i j}$ so determined are substituted in (95), that system reduces to the form

$$
\begin{array}{r}
f_{i}(x+1)=\left(a_{i}+\frac{b_{i i}}{x}\right) f_{i}(x)+\sum_{j=1}^{n}\left(\frac{c_{i j}}{x^{2}}+\cdots\right) f_{j}(x) \quad(i=1, \ldots, n) \\
|x| \geqq R^{\prime \prime} .
\end{array}
$$

Now

$$
a_{i}\left(1+\frac{1}{x}\right)^{\frac{b_{i i}}{a_{i}}}=a_{i}+\frac{b_{i i}}{x}+\cdots ;
$$

and hence, changing to more convenient notation and employing relations (81), we obtain the normal system

$$
f_{i}(x+1)-a_{i}\left(1+\frac{1}{x}\right)^{m_{i}} f_{i}(x)=\sum_{j=1}^{n} \phi_{i j}(x) f_{j}(x) \quad(i=1, \cdots, n),
$$

where

and

$$
0<\left|a_{1}\right|<\left|a_{2}\right|<\cdots<\left|a_{n}\right|, \quad m_{i}=\frac{b_{i i}}{a_{i}},
$$

$$
\phi_{i j}(x)=\phi_{i j}^{\prime \prime} x^{-2}+\phi_{i j}^{\prime \prime \prime} x^{-3}+\cdots \quad|x| \geqq R,
$$

where $R \geqq R^{\prime \prime}$. This is the normal form which we have studied in the preceding sections.

Combining the transformations (83), (85), (92) we readily obtain

$$
G_{i}(x)=\{\Gamma(x)\}^{\alpha} \sum_{k=1}^{n} \alpha_{i k}\left[f_{k}(x)+\sum_{l=1}^{n} \frac{\beta_{k l}}{x} f_{l}(x)\right] \quad(i=1, \cdots, n) .
$$

It is evident therefore that if we replace $f_{i}(x), i=1, \ldots, n$, by the particular solution $f_{i j}(x), i=1, \ldots, n, j$ fixed, we obtain a particular solution of (79) which we will call $G_{i j}(x), i=1, \ldots, n$. Doing this for $j=1, \ldots, n$, we have $n$ particular solutions

$$
G_{1 j}(x), G_{2 j}(x), \cdots, G_{n j}(x) \quad(j=1, \cdots, n),
$$


where

$$
G_{i j}(x)=\{\Gamma(x)\}^{a} \sum_{k=1}^{n} \alpha_{i k}\left[f_{k j}(x)+\sum_{l=1}^{n} \frac{\beta_{k l}}{x} f_{l j}(x)\right] \quad(i, j=1, \cdots, n) .
$$

Let us associate with (79) the corresponding equation

$$
G_{i}(x-1)=\sum_{j=1}^{n} A_{i j}(x-1) G_{j}(x) \quad(i=1, \cdots, n) .
$$

Now there is a $P$-region, as we have seen, in which the functions $f_{i j}(x)$ are al analytic. It follows from (99) that the functions $G_{i j}(x)$ likewise are all analytic in the same region. By means of (100) the functions of each of these solutions may be extended across the plane to the left; and in this way it may be shown that each of the $n^{2}$ functions $G_{i j}(x)$ is analytic throughout the entire plane except at the singularities of $A_{i j}(x)$ and the points congruent to them on the left.

Let us again write

$$
f_{i j}(x)=a_{j}^{x} x^{m_{j}} U_{i j}(x) \quad(i, j=1, \cdots, n) .
$$

If we substitute these values for $f_{i j}(x)$ in (99) we have

where

$$
G_{i j}(x)=\{\Gamma(x)\}^{a} a_{j}^{x} x^{m_{j}} V_{i j}(x), \quad(i, j=1, \cdots, n),
$$

$$
V_{i j}(x)=\sum_{k=1} \alpha_{i k}\left[U_{k j}(x)+\sum_{l=1}^{n} \frac{\beta_{k l}}{x} U_{l j}(x)\right] \text {. }
$$

From (78) it follows that when $x$ approaches infinity in the positive direction along any line parallel to the real axis we have

$$
\lim V_{i j}(x)=\alpha_{i j} \text {. }
$$

This result and the fact that the determinant $\left|\alpha_{i j}\right|$ is different from zero lead easily to the conclusion that the $n$ particular solutions $G_{i j}(x)$ are independent. The method of proof is that employed for showing the independence of the solutions $f_{i j}(x)$ in the preceding section. Hence the general solution of (79) is

$$
G_{i}(x)=\sum_{j=1}^{n} \omega_{j}(x) G_{i j}(x) \quad(i=1, \cdots, n),
$$

where the function $\omega_{j}(x), j=1, \ldots, n$, are arbitrary periodic functions of period 1.

The principal results of the section may be stated in the following theorem:

Let there be given the system of equations

where

$$
G_{i}(x+1)=x^{\alpha} \sum_{j=1}^{n} a_{i j}(x) G_{j}(x) \quad(i=1, \cdots, n),
$$

1) $\alpha$ is a constant; 
2) the functions $a_{i j}(x)$ are single-valued and

$$
a_{i j}(x)=a_{i j}+a_{i j}^{\prime} x^{-1}+a_{i j}^{\prime \prime} x^{-2}+\cdots \quad(i, j=1, \ldots, n) \quad|x|>R^{\prime} ;
$$

3) the constants $a_{i j}$ are such that the roots $\rho=a_{i}$ of the equation

$$
\left|a_{i j}-\delta_{i j} \rho\right|=0
$$

are in absolute value different from each other and from zero.

Associate with the given system the corresponding one

$$
G_{i}(x-1)=\sum_{j=1}^{n} A_{i j}(x-1) G_{j}(x) \quad(i=1, \cdots, n) .
$$

Then the given set of equations has the fundamental system of solutions

$$
G_{1 j}(x), G_{2 j}(x), \cdots, G_{n j}(x) \quad(j=1, \cdots, n),
$$

determined in the preceding pages and having the following properties : Each of the $n^{2}$ functions is analytic throughout the finite plane except at the singularities of $A_{i j}(x)$ and the points congruent to them on the left. The general solution of the system is

$$
G_{i}(x)=\sum_{j=1}^{n} \omega_{j}(x) G_{i j}(x) \quad(i=1, \cdots, n),
$$

where the functions $\omega_{j}(x), j=1, \cdots, n$, are arbitrary periodic functions of period 1. Further, if $x$ approaches infinity in the positive direction along any line parallel to the real axis we have

$$
\lim G_{i j}(x)\{\Gamma(x)\}^{-a} a_{j}^{-x} x^{-m_{j}}=\alpha_{i j},
$$

where the constants $\alpha_{i j}$ are determined by equations (90) and the constants $m_{j}$ are those introduced in (97).

Let us now consider the single difference equation of the $n$th order

$$
\begin{aligned}
& H(x+n)+(x+n)^{a} b_{1}(x) H(x+n-1)+(x+n)^{a}(x+n-1)^{a} \\
& \quad \times b_{2}(x) H(x+n-2)+\cdots+(x+n)^{a} \cdots(x+1)^{a} x^{a} b_{n}(x) H(x)=0
\end{aligned}
$$

where $\alpha$ is a constant and $b_{i}(x)$ is single-valued and

$$
b_{i}(x)=b_{i}+b_{i}^{\prime} x^{-1}+b_{i}^{\prime \prime} x^{-2}+\cdots \quad(i=1, \cdots, n) \quad|x|>R,
$$

and where the constants $b_{i}$ are such that the roots $a_{1}, \ldots, a_{n}$ of the equation

$$
\rho^{n}+b_{1} \rho^{n-1}+b_{2} \rho^{n-2}+\cdots+b_{n-1} \rho+b_{n}=0
$$

are in absolute value different from each other and from zero.

Making the substitution

$$
H(x)=\{\Gamma(x)\}^{a} \bar{H}(x)
$$


we have

(104) $\bar{H}(x+n)+b_{1}(x) \bar{H}(x+n-1)+\cdots+b_{n}(x) \bar{H}(x)=0$.

It is evident that this equation is equivalent to the following system

where

$$
\begin{array}{ll}
\bar{H}_{1}(x+1) & =\sum_{j=1}^{n}\left\{-b_{j}(x)\right\} \bar{H}_{j}(x)=0, \\
\bar{H}_{i}(x+1) & =\bar{H}_{i-1}(x)
\end{array}
$$

$$
\bar{H}_{n}(x) \equiv \bar{H}(x)
$$

Now the characteristic equation [compare (80)] associated with (105) is readily reduced to the form (102).

If we solve $(105)$ for $\bar{H}_{i}(x)$ in terms of $\bar{H}_{i}(x+1), i=1, \ldots, n$, and in the result write $x-1$ for $x$, we have

$$
\begin{gathered}
\bar{H}_{i}(x-1)=\bar{H}_{i+1}(x) \quad(i=1, \cdots, n-1), \\
\bar{H}_{n}(x-1)=-\frac{1}{b_{n}(x-1)} \bar{H}_{1}(x)-\sum_{j=1}^{n-1} \frac{b_{j}(x-1)}{b_{n}(x-1)} \bar{H}_{j+1}(x) .
\end{gathered}
$$

Consequently, from the foregoing theorem, we have the following conclusions : The system (105) has a fundamental system of solutions

$$
\bar{H}_{1 j}(x), \bar{H}_{2 j}(x), \cdots, \bar{H}_{n j}(x) \quad(j=1, \cdots, n),
$$

each function of which is analytic throughout the finite plane except at the singularities of the functions

$$
\frac{1}{b_{n}(x)}, \frac{b_{j}(x)}{b_{n}(x)} \quad(j=1, \cdots, n),
$$

and points congruent to them on the left; further, if $x$ approaches infinity in the positive direction along any line parallel to the real axis, we have

$$
\lim \bar{H}_{n j}(x) a_{j}^{-x} x^{-m_{j}}=\bar{\alpha}_{i j},
$$

where $\bar{\alpha}_{i j}$ and $m_{j}$ are constants corresponding to the $\alpha_{i j}$ and $m_{j}$ of the above theorem. Since only the ratios of $\bar{\alpha}_{i j}, j$ fixed, $i=1, \cdots, n$, are determined, we take $\bar{\alpha}_{n j}=1, j=1, \cdots, n$.

Combining (103) and (106) we have

$$
H(x)=\{\Gamma(x)\}^{\alpha} \bar{H}_{n}(x)
$$

Hence we have a fundamental system of solutions $H_{j}(x), j=1, \ldots, n$, of (101) in the form

$$
H_{j}(x)=\{\Gamma(x)\}^{a} \bar{H}_{n j}(x) \quad(j=1, \cdots, n) .
$$


Making use of this equation and the properties of $\bar{H}_{n j}(x)$ stated above and determining the position of the singularities from the equation itself by extending the solutions across the plane to the left, we obtain the results stated in the conclusion of the following theorem :

Given, the difference equation

$$
\begin{gathered}
H(x+n)+(x+n)^{a} b_{1}(x) H(x+n-1)+\cdots+(x+n)^{a} \cdots(x+1)^{a} x^{a} b_{n}(x) H(x)=0 \\
o r \quad \beta_{n}(x) H(x+n)+\beta_{n-1}(x) H(x+n-1)+\cdots+\beta_{1}(x) H(x+1)+H(x)=0,
\end{gathered}
$$

where $\alpha$ is a constant and $b_{i}(x)$ is single-valued and

$$
b_{i}(x)=b_{i}+b_{i}^{\prime} x^{-1}+b_{i}^{\prime \prime} x^{-2}+\cdots \quad(i=1, \ldots, n) \quad|x|>R,
$$

and where the constants $b_{i}$ are such that the roots $a_{1}, \ldots, a_{n}$ of the equation

$$
\rho^{n}+b_{1} \rho^{n-1}+\cdots+b_{n-1} \rho+b_{n}=0
$$

are in absolute value different from each other and from zero.

This equation has the fundamental system of solutions

$$
H_{1}(x), H_{2}(x), \ldots, H_{n}(x)
$$

determined above and possessing the follouing properties: Each of the functions $H_{1}(x), \ldots, H_{n}(x)$ is analytic throughout the finite plane except at the. singularities of the functions $\beta_{i}(x), i=1, \ldots, n$, and the points congruent to them on the left. If $x$ approaches infinity in the positive direction along any line parallel to the real axis, we have

$$
\lim H_{j}(x)\{\Gamma(x)\}^{-a} a_{j}^{-x} x^{-m_{j}}=1 \quad(j=1, \cdots, n),
$$

where $m_{j}$ are constants.

The general solution of the eqation is

$$
H(x)=\omega_{1}(x) H_{1}(x)+\cdots+\omega_{n}(x) H_{n}(x)
$$

where $\omega_{1}(x), \cdots, \omega_{n}(x)$ are arbitrary periodic functions of period 1 .

It may be observed that a modification of the foregoing method will lead to a different fundamental system of solutions of (79) and likewise of (101). We confine our attention to (79). A few remarks relative to the equation

$$
g(x+1)-a\left(1+\frac{1}{x}\right)^{m} g(x)=\eta(x)
$$

will sufficiently indicate the necessary changes. Employing the notation

$$
\lambda(x) \equiv a\left(1+\frac{1}{x}\right)^{m}
$$


we have two formal solutions of the above equation as follows:

$$
\begin{aligned}
& g(x)=-\sum_{i=0}^{\infty} \frac{\eta(x+i)}{\lambda(x) \lambda(x+1) \cdots \lambda(x+i)}, \\
& g(x)=\eta(x-1)+\sum_{i=1}^{\infty} \lambda(x-1) \lambda(x-2) \cdots \lambda(x-i) \eta(x-i-1) .
\end{aligned}
$$

The first of these, as we have seen, leads to a solution of (1). The second may also be employed in a similar way to obtain a solution of the same equation. The extension to a system of $n$ equations is obvious. In this way a second fundamental system of solutions of (79) may be found.

Suppose further that transformation (83) is modified by the use of a function $\bar{\Gamma}(x)$ which satisfies the equation

$$
\bar{\Gamma}(x+1)=x \bar{\Gamma}(x)
$$

and is analytic to the left of the axis of imaginaries, this function replacing $\Gamma(x)$ in (83). This modification having been made, it will turn out that each function of the fundamental system just found is analytic throughout the finite plane except at the singularities of $a_{i j}(x)$ and the points congruent to them on the right; and their properties may be developed by a method similar throughout to that employed in the paper and with like results.

Princeton Univeritit, August, 1910. 